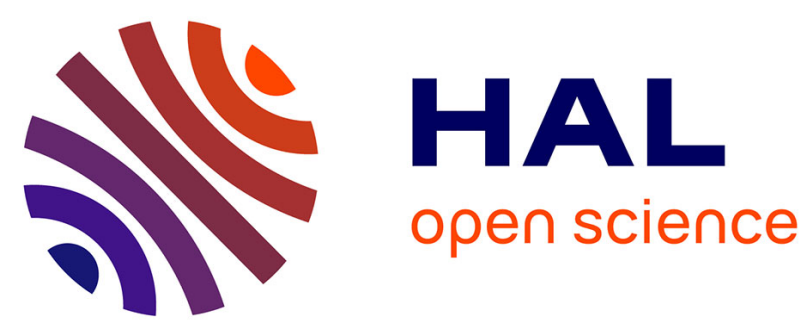

\title{
Double overreaction in beauty-contests with information acquisition: theory and experiment
}

\author{
Romain Baeriswyl, Kene Boun My, Camille Cornand
}

\section{To cite this version:}

Romain Baeriswyl, Kene Boun My, Camille Cornand. Double overreaction in beauty-contests with information acquisition: theory and experiment. Journal of Monetary Economics, 2021, 118, pp.432445. 10.1016/j.jmoneco.2020.12.004 . hal-03468857

\section{HAL Id: hal-03468857 https://hal.science/hal-03468857}

Submitted on 7 Dec 2021

HAL is a multi-disciplinary open access archive for the deposit and dissemination of scientific research documents, whether they are published or not. The documents may come from teaching and research institutions in France or abroad, or from public or private research centers.
L'archive ouverte pluridisciplinaire HAL, est destinée au dépôt et à la diffusion de documents scientifiques de niveau recherche, publiés ou non, émanant des établissements d'enseignement et de recherche français ou étrangers, des laboratoires publics ou privés. 
UMR 5824

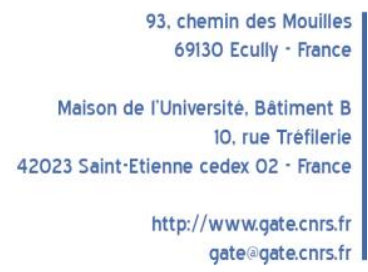

WP 1932 - November 2019, revised February 2020

\title{
Double overreaction in beauty-contests with information acquisition: theory and experiment
}

\author{
Romain Baeriswyl, Kene Boun My, Camille Cornand
}

\begin{abstract}
:
Central banks' disclosures, such as forward guidance, have a weaker effect on the economy in reality than in theoretical models. The present paper contributes to understanding how people pay attention and react to various sources of information. In a beauty-contest with information acquisition, we show that strategic complementarities give rise to a double overreaction to public disclosures by increasing agents equilibrium attention, which, in turn, increases the weight assigned to them in equilibrium action. A laboratory experiment provides evidence that the effect of strategic complementarities on the realised attention and the realised action is qualitatively consistent with theoretical predictions, though quantitatively weaker. Both the lack of attention to public disclosures and a limited level of reasoning by economic agents account for the weaker realised reaction. This suggests that it is just as important for a central bank to control reaction to public disclosures by swaying information acquisition by recipients as it is by shaping information disclosures themselves.
\end{abstract}

\section{Keywords:}

beauty-contest, information acquisition, overreaction, central bank communication

JEL codes:

D82, E52, E58

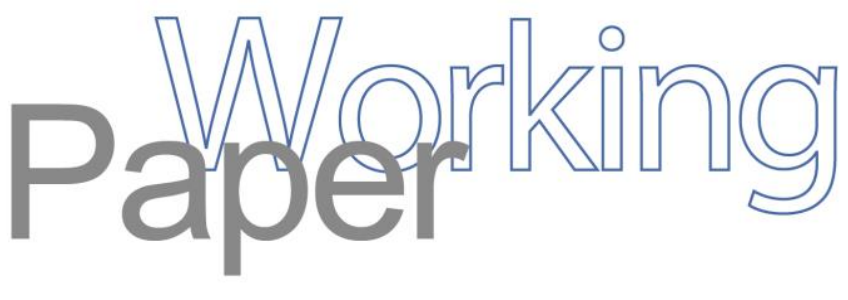




\title{
Double overreaction in beauty contests with information acquisition: theory and experiment*
}

\author{
Romain Baeriswyl ${ }^{\S}$ Kene Boun $\mathrm{My}^{\sharp} \quad$ Camille Cornand ${ }^{\dagger}$
}

February 2020

\begin{abstract}
Central banks' disclosures, such as forward guidance, have a weaker effect on the economy in reality than that predicted in theoretical models. The present paper contributes to understanding how people pay attention and react to various sources of information. In a beauty contest with information acquisition, we show that strategic complementarities give rise to a double overreaction to public disclosures by increasing agents equilibrium level of attention, which, in turn, increases the weight assigned to the disclosures in agents' equilibrium action. A laboratory experiment provides evidence that the effect of strategic complementarities on participants' realised level of attention and realised action is qualitatively consistent with the theoretical predictions, although quantitatively weaker. Both the lack of attention to public disclosures and a limited level of reasoning by economic agents account for the weaker realised reaction. This suggests that for a central bank seeking to control the reaction to its public disclosures, it is just as important to influence information acquisition by recipients as it is to shape the information disclosures.
\end{abstract}

JEL classification: D82, E52, E58.

Keywords: beauty contest, information acquisition, overreaction, central bank communication.

${ }^{*}$ This research has benefited from the financial support of IDEXLYON from Université de Lyon (project INDEPTH) within the Programme Investissements d'Avenir (ANR-16-IDEX-0005) and it was performed within the framework of the LABEX CORTEX (ANR-11-LABX-0042) of Université de Lyon, within the program Investissements d'Avenir (ANR-11-IDEX-007) operated by the French National Research Agency. The views expressed in this paper are strictly those of the authors and do not necessarily reflect those of the Swiss National Bank.

${ }^{\S}$ Swiss National Bank, Boersenstrasse 15, 8001 Zurich, Switzerland; email: romain.baeriswyl@snb.ch

${ }^{\sharp}$ BETA-Strasbourg University, 61 Avenue de la Forêt Noire, F-67085 Strasbourg, France; email: bounmy@unistra.fr

${ }^{\dagger}$ Univ Lyon, CNRS, GATE UMR 5824, F-69130 Ecully, France; email: cornand@gate.cnrs.fr 


\section{Introduction}

The role of central bank communication in the conduct of monetary policy has increased considerably in recent decades. While communication is essential for establishing the democratic legitimacy of an independent central bank, it has increasingly been used as a monetary policy instrument for managing market participants' expectations. As pointed out by Woodford (2005, p.3), "for [monetary policy to be most effective] not only do expectations about policy matter, but, at least under current conditions, very little else matters." Forward guidance is perhaps the best example of an instrument for managing expectations. By disclosing information on the policy it intends to pursue, the central bank aims to influence market expectations of future policy rates and thereby long-term interest rates and inflation expectations.

The coordinating role of public information has received considerable attention in the ongoing debate on how a central bank should communicate. In environments characterised by strategic complementarities, such as financial markets or price setting under monopolistic competition, economic agents seek to coordinate their actions while remaining close to economic fundamentals. When it is common knowledge, public information reduces uncertainty about others' information and thereby enhances coordination among economic agents. However, despite its prominence in theory, common knowledge is difficult to achieve in reality. Common knowledge not only requires that a sender disclose public information but also that receivers pay attention to public information and that they know that all other receivers pay attention to the same public information as well, up to an infinite level of iteration. Thus, understanding how agents pay attention to various sources of information is essential for anticipating their reaction and for designing an optimal communication policy.

This paper contributes to understanding how people pay attention to various sources of information when they face strategic complementarities. First, we provide a theory of a double overreaction to the most common and least private signal in a beauty contest with information acquisition. On the one hand, strategic complementarities increase the equilibrium weight assigned to the most common and least private signal because it helps to coordinate with other agents. This is the overreaction mechanism described by Morris and Shin (2002). On the other hand, strategic complementarities also increase the equilibrium attention given to the most common and least private signal, which further increases its equilibrium weight in the beauty contest.

Second, in a laboratory experiment, we investigate the double overreaction mechanism with three treatments. Treatment A implements the beauty contest with exogenous information to control for the overreaction to public information. Treatment B implements the beauty contest with information acquisition, where it is optimal to pay full attention to the most common and least private signal. Treatment $\mathrm{C}$ implements a fundamental-estimation game with information acquisition, where it is optimal to pay no attention to the most common and least private signal. In response to strategic complementarities, participants increase both the attention given and the weight assigned to the most common and least 
private signal, confirming the double overreaction mechanism predicted by theory. Moreover, in accordance with theory, participants tend to assign a higher weight to the most common and least private signal as they pay more attention to it. The overreaction, however, is weaker than theoretically predicted.

Based on participants' elicited beliefs, we break down deviations from the equilibrium of the realised weight in the beauty contest into four possible sources of error, namely, a suboptimal allocation of attention, a suboptimal expectation of the fundamental, a suboptimal expectation of the average action of others, and a suboptimal action in view of the elicited expectations. Deviations from equilibrium are mainly explained by a suboptimally weak effect of strategic complementarities on both the allocation of attention and the expectations of the other participants' actions. Deviations from equilibrium in terms of both attention and action can be measured in terms of limited levels of reasoning. These limited levels of reasoning are heterogeneous among subjects and are mainly distributed between level 0 and level 2 .

The experiment highlights the role of information acquisition in the reaction of participants to public disclosures. This finding provides some insight into the so-called forward guidance puzzle documented by Del Negro et al. (2015), which refers to the surprisingly weak reaction of market participants to central banks' disclosures about the banks' intended future policy compared to the reactions predicted theoretically in standard new Keynesian models. Shaping market expectations seems more challenging in practice than in theory. Angeletos and Lian (2018) propose the lack of common knowledge and GarciaSchmidt and Woodford (2018) the limited level of reasoning as possible causes of this puzzle. Both hypotheses are supported by our experiment: a limited level of reasoning explains weak attention to the most common and least private signal; this weak attention then prevents the emergence of common knowledge among participants. Moreover, given their information, participants typically deploy a limited level of reasoning in choosing their action.

Section 2 discusses the related literature, while section 3 presents the model and section 4 the experimental setup. Section 5 presents the experimental results, and section 6 concludes.

\section{Related literature}

Our paper relates to the literature on information acquisition in coordination environments under heterogeneous information. Different approaches have been proposed to account for the fact that to be effective, information must not only be disclosed by an issuer but also observed and processed by recipients. A general result from models with a continuum of actions and a quadratic payoff structure (mimicking the business cycle type of environment) is that strategic complementarities in actions imply that the acquisition of information is characterised by strategic complementarities as well. In Hellwig and Veldkamp (2009), a binary allocation of attention to information sources with exogenous 
publicity results in equilibrium indeterminacy. By contrast, in Myatt and Wallace (2012), agents can acquire different signals at some cost, so that the publicity of each information source is an equilibrium phenomenon. The more agents' actions are complementary, the more the information acquired becomes public. Our model differs from that of Myatt and Wallace in that, rather than introducing observation costs, agents allocate their limited information-processing resources among different sources of information. Myatt and Wallace (2014) provide an application to a Lucas-Phelps island economy. Pavan (2016) - distinguishing between attention and recall - adopts the same information structure as Myatt and Wallace but proposes a more general payoff structure allowing him to address normative questions, as in Angeletos and Pavan (2007) and Colombo et al. (2014). Focusing on optimal central bank disclosure, Chahrour (2014) investigates agents' miscoordination depending on the source of costly information they pay attention to. Llosa and Venkateswaran (2013), on the other hand, compare the equilibrium vs. efficient acquisition of information in different specifications of the business cycle.

Our paper also belongs to the experimental literature on the beauty contest game under heterogeneous information, formalised by Morris and Shin (2002). Considering the original beauty contest with an exogenous information structure, Cornand and Heinemann (2014) experimentally study agents' overreaction to public information. Baeriswyl and Cornand (2014) show that partial publicity (consisting of providing a public signal to a subset of agents only) and partial transparency (consisting of providing a public signal with idiosyncratic noise) may effectively reduce overreaction to public disclosures. To our knowledge, the present paper is the first to study information acquisition in an experimental beauty contest.

While the literature mentioned above considers economies with a continuum of actions and continuous payoffs, information acquisition in games of regime change (with discontinuous payoffs and binary actions) is studied experimentally by Szkup and Trevino (2017). They show that the endogenous determination of the information structure by subjects (i.e., agents choose at a cost the precision of their private signal) affects the standard results in terms of information precision comparative statics and improves the efficiency of the actions. In contrast to the latter, the quadratic payoff structure of our study presents the advantage of having many applications in the business cycle literature.

Finally, the present paper is related to the literature on rational inattention (see, e.g., Sims (2003) for a pioneering work and Mackowiak and Wiederholt (2015) for a business cycle application). Recurring to information theory and focusing on receiver (rather than sender) noise, this literature shows that agents may rationally neglect information when information acquisition and processing are costly and the expected returns are small. This literature also offers an experimental counterpart. For example, in an experimental study, Cheremukhin et al. (2015) estimate and compare different models of rational inattention and show that subjects have heterogeneous costs of information processing. Caplin and Dean (2015) provide evidence that subjects collect more information, use more time and put more effort into processing information if the rewards for doing so are higher. Caplin 
and Dean (2013) emphasise, however, that subjects respond less to changes in payoff incentives than the Shannon entropy theory predicts.

\section{The theoretical model}

The problem of information acquisition is derived from a quadratic payoff beauty contest game. The economy is populated by a continuum of agents indexed by the unit interval $i \in[0,1]$. To maximise its utility, each agent $i$ chooses an action that is close to both an unknown fundamental and the average action of other agents $-i$. Each agent also chooses how much attention she pays to the two available sources of information. An agent's strategy consists of choosing two weights (between 0 and 1), one determining the allocation of her information-processing resources between the two sources of information and the other determining the weight of the two signals in her beauty contest action.

\subsection{A beauty contest game}

The utility of agent $i$ decreases in both the distortion of her action $a_{i}$ from the unknown fundamental $\theta \in \mathbb{R}$ and the dispersion of her action from the average action of other agents $\bar{a}_{-i}$

$$
u_{i}(a, \theta)=\bar{u}-(1-\gamma)\left(a_{i}-\theta\right)^{2}-\gamma\left(a_{i}-\bar{a}_{-i}\right)^{2}
$$

The parameter $\gamma$ is the weight assigned to the strategic component that drives the strength of the coordination motive in the utility function and decision rule. If $\gamma=0$, agents' utility is independent of the dispersion of their action, and agents take their action to be as close as possible to the fundamental $\theta$. If $0<\gamma<1$, agents' actions are strategic complements: agents tend to align their action with those of others.

\subsection{Information sources}

Agents have access to two information sources, $x_{1}$ and $x_{2}$, on the unknown fundamental. Each of these information sources contains an error term that is common to all agents and that is normally distributed with a zero mean and some variance

$$
\begin{array}{ll}
x_{1}=\theta+\eta_{1} & \text { where } \eta_{1} \sim N\left(0, \kappa_{1}^{2}\right) \\
x_{2}=\theta+\eta_{2} & \text { where } \eta_{2} \sim N\left(0, \kappa_{2}^{2}\right)
\end{array}
$$

However, agents do not directly observe the information sources $x_{1}$ and $x_{2}$. Instead, they observe these information sources with some idiosyncratic noise, which depends on the attention given to each source. Each agent has a total amount of attention of 1, which she allocates between the two information sources. The total attention can be interpreted as the time available to an agent for processing information. The information-acquisition problem of agent $i$ consists of choosing the share of her total attention $z_{i} \in[0,1]$ devoted 
to observing the information source $x_{1}$. The residual attention of agent $i, 1-z_{i}$, is devoted to observing the information source $x_{2}$. Assuming that attention additively reduces the variance of the idiosyncratic noise of observing the information sources, the two signals received by agent $i$ are characterised by the following noise terms

$$
\begin{array}{ll}
x_{1, i}=x_{1}+\epsilon_{1, i} & \text { where } \epsilon_{1, i} \sim N\left(0, \xi_{1}^{2}+1-z_{i}\right) \\
x_{2, i}=x_{2}+\epsilon_{2, i} & \text { where } \epsilon_{2, i} \sim N\left(0, \xi_{2}^{2}+z_{i}\right)
\end{array}
$$

where all noise terms are independently distributed.

The variance of the signals has a component common to all agents, $\kappa_{1}^{2}$ and $\kappa_{2}^{2}$, that can be interpreted as the accuracy (precision) of the source or as the sender noise, and a component specific to each agent, $\xi_{1}^{2}+1-z_{i}$ and $\xi_{2}^{2}+z_{i}$, that can be interpreted as the transparency (clarity) of the source or as the receiver noise. The receiver noise terms are specific to each agent (i) because they are drawn independently for each agent from a normal distribution and (ii) because each agent, by choosing $z_{i}$, shapes the variance of the normal distribution from which they are drawn.

\subsection{Equilibrium}

An equilibrium strategy for agent $i$ is a set $\left\{z_{i}, \omega_{i}\right\}$ that specifies her attention and action decision as a function of the model parameters. While $z_{i}$ determines the allocation of attention between information sources, $\omega_{i}$ determines the relative weight of each signal in the agent's action.

\subsubsection{Action}

We first derive the equilibrium action for a given level of attention and show that the equilibrium action is optimal from the agent's perspective. Then, we derive the equilibrium attention allocation.

Equilibrium action The action of agent $i$ that maximises her utility (1) is given by the first-order condition

$$
a_{i}=(1-\gamma) \mathbb{E}_{i}(\theta)+\gamma \cdot \mathbb{E}_{i}\left(\bar{a}_{-i}\right)
$$

where $\mathbb{E}_{i}$ is the expectation operator conditional on the information of agent $i$. To derive agent $i$ 's perfect Bayesian equilibrium action, $a_{i}$, we compute the expectation of the fundamental $\theta$ and the expectation of others' action $\bar{a}_{-i}$. Based on her signals, the expectation of agent $i$ about the fundamental $\theta$ is given by

$$
\mathbb{E}_{i}(\theta)=\underbrace{\frac{\kappa_{2}^{2}+\xi_{2}^{2}+z_{i}}{\kappa_{1}^{2}+\kappa_{2}^{2}+\xi_{1}^{2}+\xi_{2}^{2}+1}}_{f} x_{1, i}+\underbrace{\frac{\kappa_{1}^{2}+\xi_{1}^{2}+1-z_{i}}{\kappa_{1}^{2}+\kappa_{2}^{2}+\xi_{1}^{2}+\xi_{2}^{2}+1}}_{1-f} x_{2, i}
$$


To derive her expectation of others' action, we need to express her expectation about others' average information $\bar{x}_{1}$ and $\bar{x}_{2}$. The expected average signals are

$$
\begin{aligned}
& \mathbb{E}_{i}\left(\bar{x}_{1}\right)=\underbrace{\frac{\kappa_{1}^{2}+\kappa_{2}^{2}+\xi_{2}^{2}+z_{i}}{\kappa_{1}^{2}+\kappa_{2}^{2}+\xi_{1}^{2}+\xi_{2}^{2}+1}}_{\Omega_{1}} x_{1, i}+\underbrace{\frac{\xi_{1}^{2}+1-z_{i}}{\kappa_{1}^{2}+\kappa_{2}^{2}+\xi_{1}^{2}+\xi_{2}^{2}+1}}_{\Omega_{2}} x_{2, i} \\
& \mathbb{E}_{i}\left(\bar{x}_{2}\right)=\underbrace{\frac{\xi_{2}^{2}+z_{i}}{\kappa_{1}^{2}+\kappa_{2}^{2}+\xi_{1}^{2}+\xi_{2}^{2}+1}}_{1-\Omega_{1}} x_{1, i}+\underbrace{\frac{\kappa_{1}^{2}+\kappa_{2}^{2}+\xi_{1}^{2}+1-z_{i}}{\kappa_{1}^{2}+\kappa_{2}^{2}+\xi_{1}^{2}+\xi_{2}^{2}+1}}_{\Omega_{1}} x_{2, i}
\end{aligned}
$$

Following Morris and Shin (2002), let us assume that the equilibrium action is a linear combination of signals

$$
a_{i}=\omega_{i} \cdot x_{1, i}+\left(1-\omega_{i}\right) x_{2, i}
$$

where $\omega_{i}$ is the weight assigned to signal $x_{1, i}$ and $1-\omega_{i}$ is the weight assigned to signal $x_{2, i}$ in the beauty contest action. Because all agents are identical and face the same problem, agent $i$ rationally assumes that other agents choose the same attention allocation $z_{i}$ and the same weight in action $\omega_{i}$ as she does. Her expectation of others' average action $\bar{a}_{-i}$ is

$$
\mathbb{E}_{i}\left(\bar{a}_{-i}\right)=\underbrace{\left(\omega_{i} \cdot \Omega_{1}+\left(1-\omega_{i}\right) \Omega_{2}\right)}_{o} x_{1, i}+\underbrace{\left(\omega_{i}\left(1-\Omega_{1}\right)+\left(1-\omega_{i}\right)\left(1-\Omega_{2}\right)\right)}_{1-o} x_{2, i}
$$

Given the expectations of the fundamental and of others' action, the equilibrium action can be expressed as

$$
\begin{aligned}
a_{i} & =(1-\gamma)\left(f \cdot x_{1, i}+(1-f) x_{2, i}\right)+\gamma\left(o \cdot x_{1, i}+(1-o) x_{2, i}\right) \\
& =((1-\gamma) f+\gamma \cdot o) x_{1, i}+((1-\gamma)(1-f)+\gamma(1-o)) x_{2, i} \\
& =\frac{(1-\gamma) \kappa_{2}^{2}+\xi_{2}^{2}+z_{i}}{(1-\gamma)\left(\kappa_{1}^{2}+\kappa_{2}^{2}\right)+\xi_{1}^{2}+\xi_{2}^{2}+1} x_{1, i}+\frac{(1-\gamma) \kappa_{1}^{2}+\xi_{1}^{2}+1-z_{i}}{(1-\gamma)\left(\kappa_{1}^{2}+\kappa_{2}^{2}\right)+\xi_{1}^{2}+\xi_{2}^{2}+1} x_{2, i} \\
& =\underbrace{\frac{\nu_{2}+z_{i}}{\nu_{1}+\nu_{2}+1}}_{\omega_{i}} x_{1, i}+\underbrace{\frac{\nu_{1}+1-z_{i}}{\nu_{1}+\nu_{2}+1}}_{1-\omega_{i}} x_{2, i}
\end{aligned}
$$

where $\nu_{1}=(1-\gamma) \kappa_{1}^{2}+\xi_{1}^{2}$ and $\nu_{2}=(1-\gamma) \kappa_{2}^{2}+\xi_{2}^{2}$.

Optimal action The equilibrium action (4) is optimal in the sense that it maximises agents' utility. With $\bar{\omega}$ being defined as the average weight $\int_{i} \omega_{i} d i$ over all agents, the unconditional expected utility yields

$$
\begin{aligned}
\mathbb{E}\left(u_{i}\right)= & \bar{u}-(1-\gamma) \mathbb{E}\left(\omega_{i} \cdot x_{1, i}+\left(1-\omega_{i}\right) x_{2, i}-\theta\right)^{2} \\
& -\gamma \mathbb{E}\left(\omega_{i} \cdot x_{1, i}+\left(1-\omega_{i}\right) x_{2, i}-\bar{\omega} \cdot \bar{x}_{1}-(1-\bar{\omega}) \bar{x}_{2}\right)^{2} \\
= & \bar{u}-\omega_{i}^{2}\left[\nu_{1}+1-z_{i}\right]-\left(1-\omega_{i}\right)^{2}\left[\nu_{2}+z_{i}\right]-\gamma\left(\omega_{i}-\bar{\omega}\right)^{2}\left[\kappa_{1}^{2}+\kappa_{2}^{2}\right]
\end{aligned}
$$


Differentiating (5) with respect to $\omega_{i}$ yields

$$
\begin{aligned}
\frac{\partial \mathbb{E}\left(u_{i}\right)}{\partial \omega_{i}} & =-2 \omega_{i}\left(\nu_{1}+1-z_{i}\right)+2\left(1-\omega_{i}\right)\left(\nu_{2}+z_{i}\right)-2 \gamma\left(\omega_{i}-\bar{\omega}\right)\left(\kappa_{1}^{2}+\kappa_{2}^{2}\right) \\
& =0 \Leftrightarrow \omega_{i}=\frac{\nu_{2}+z_{i}+\gamma \bar{\omega}\left(\kappa_{1}^{2}+\kappa_{2}^{2}\right)}{\nu_{1}+\nu_{2}+1+\gamma\left(\kappa_{1}^{2}+\kappa_{2}^{2}\right)} \\
\frac{\partial \omega_{i}}{\partial \bar{\omega}} & =\frac{\gamma\left(\kappa_{1}^{2}+\kappa_{2}^{2}\right)}{\nu_{1}+\nu_{2}+1+\gamma\left(\kappa_{1}^{2}+\kappa_{2}^{2}\right)}<1 \\
\frac{\partial^{2} \mathbb{E}\left(u_{i}\right)}{\partial \omega_{i} \partial z_{i}} & =2>0
\end{aligned}
$$

Equation (6) shows that the optimal action of agent $i$ increases with her attention allocation $z_{i}$ and with others' average action $\bar{\omega}$. Since agents are all identical and the slope of the best response to others' action (7) is smaller than one, in the optimal equilibrium, $\omega_{i}=\bar{\omega}$, and the weight (6) simplifies to

$$
\omega_{i}=\frac{\nu_{2}+z_{i}}{\nu_{1}+\nu_{2}+1}=\frac{(1-\gamma) \kappa_{2}^{2}+\xi_{2}^{2}+z_{i}}{(1-\gamma)\left(\kappa_{1}^{2}+\kappa_{2}^{2}\right)+\xi_{1}^{2}+\xi_{2}^{2}+1}
$$

which is equivalent to the equilibrium action (4). The positive cross partial derivative (8) indicates that the optimal attention level $z_{i}$ and the optimal weight $\omega_{i}$ are complements.

\subsubsection{Attention}

Having derived the equilibrium action, we turn to the equilibrium attention. Differentiating utility (5) with respect to $z_{i}$ yields the following first-order condition

$$
\frac{\partial \mathbb{E}\left(u_{i}\right)}{\partial z_{i}}=2 \omega_{i}-1 \lesseqgtr 0 \quad \Leftrightarrow \quad \omega_{i} \lesseqgtr \frac{1}{2}
$$

Increasing the attention given to the signal $x_{1, i}$ improves utility when the weight assigned to it in the decision $a_{i}, \omega_{i}$, is greater than $1 / 2$. Because $z \in[0,1]$, optimal attention is either 0 or 1 , and it is never optimal to choose an intermediate level of attention unless $\omega_{i}=1 / 2$, in which case the optimal attention is indeterminate. It is thus optimal to pay full attention to the signal that is highest weighted in the action.

With this result, optimal attention is obtained by comparing the unconditional expected utility with zero vs. full attention paid to $x_{1, i}$, assuming that agents choose the action that is optimal for the given attention allocation. Then we check whether optimal attention is also an equilibrium and whether it is the only equilibrium. It turns out that while optimal attention is always an equilibrium, a suboptimal equilibrium attention may appear depending on the parameter values. ${ }^{1}$

Optimal attention When all agents choose the same attention allocation and the same weight in action, $z_{i}=z$ and $\omega_{i}=\omega \forall i$, the expected utility with zero attention paid to

\footnotetext{
${ }^{1}$ It should be noted that, given a certain attention decision, the equilibrium action is unique, as shown in Morris and Shin (2002). The possibility of multiple equilibria arises only in terms of attention.
} 
$x_{1, i},\left.\mathbb{E}\left(u_{i}\right)\right|_{z=0}$, is higher than expected utility with full attention paid to $x_{1, i},\left.\mathbb{E}\left(u_{i}\right)\right|_{z=1}$, when the following condition holds

$$
\begin{aligned}
\left.\mathbb{E}\left(u_{i}\right)\right|_{z=0}>\left.\mathbb{E}\left(u_{i}\right)\right|_{z=1} & \Leftrightarrow \nu_{1}>\nu_{2} \\
z^{*} & =0 \\
\omega^{*} & =\frac{\nu_{2}}{\nu_{1}+\nu_{2}+1} \\
\mathbb{E}\left(u_{i}\right) & =-\frac{\left(\nu_{1}+1\right) \nu_{2}}{\nu_{1}+\nu_{2}+1}
\end{aligned}
$$

In the opposite case, the expected utility with full attention paid to $x_{1, i},\left.\mathbb{E}\left(u_{i}\right)\right|_{z=1}$, is higher than the expected utility with zero attention paid to $x_{1, i},\left.\mathbb{E}\left(u_{i}\right)\right|_{z=0}$, when

$$
\begin{aligned}
\left.\mathbb{E}\left(u_{i}\right)\right|_{z=0}<\left.\mathbb{E}\left(u_{i}\right)\right|_{z=1} & \Leftrightarrow \nu_{1}<\nu_{2} \\
z^{*} & =1 \\
\omega^{*} & =\frac{\nu_{2}+1}{\nu_{1}+\nu_{2}+1} \\
\mathbb{E}\left(u_{i}\right) & =-\frac{\nu_{1}\left(\nu_{2}+1\right)}{\nu_{1}+\nu_{2}+1}
\end{aligned}
$$

Conditions (11) and (12) show that it is optimal to pay full attention to the signal with the lowest adjusted variance of error terms $(1-\gamma) \kappa^{2}+\xi^{2}$. Without strategic complementarities, i.e., when $\gamma=0$, full attention should be given to the signal with the lowest variance of error terms $\kappa^{2}+\xi^{2}$. With increasing strategic complementarities, the variance of common error terms becomes relatively less detrimental to utility than the variance of private error terms because the former enhances coordination. Attention is thus to be given to the most common and least private signal.

Equilibrium attention To check if optimal attention is an equilibrium, we compare the expected utility (5) when agent $i$ plays the optimal strategy $\left\{z_{i}^{*}, \omega_{i}^{*}\right\}$ given by (11) or (12) while other agents play the suboptimal strategy $\left\{\tilde{z}_{-i}, \omega\left(\tilde{z}_{-i}\right)\right\}$

$$
\left.\mathbb{E}\left(u_{i}\right)\right|_{z_{i}^{*}, \tilde{z}_{-i}}=\bar{u}-\omega_{i}^{* 2}\left[\nu_{1}+1-z_{i}^{*}\right]-\left(1-\omega_{i}^{*}\right)^{2}\left[\nu_{2}+z_{i}^{*}\right]-\gamma\left(\omega_{i}^{*}-\omega\left(\tilde{z}_{-i}\right)\right)^{2}\left[\kappa_{1}^{2}+\kappa_{2}^{2}\right]
$$

with the expected utility when agent $i$ plays the same suboptimal strategy $\left\{\tilde{z}_{-i}, \omega\left(\tilde{z}_{-i}\right)\right\}$ as other agents

$$
\left.\mathbb{E}\left(u_{i}\right)\right|_{z_{i}=\tilde{z}_{-i}}=\bar{u}-\omega\left(\tilde{z}_{-i}\right)^{2}\left[\nu_{1}+1-\tilde{z}_{-i}\right]-\left(1-\omega\left(\tilde{z}_{-i}\right)\right)^{2}\left[\nu_{2}+\tilde{z}_{-i}\right]
$$

When $\nu_{1}>\nu_{2}$, the optimal strategy (11) with $z^{*}=0$ yields a higher utility to agent $i$ than the utility from the suboptimal strategy $\tilde{z}_{-i}$ when

$$
\left.\mathbb{E}\left(u_{i}\right)\right|_{z_{i}^{*}=0, \tilde{z}_{-i}}>\left.\mathbb{E}\left(u_{i}\right)\right|_{z_{i}=\tilde{z}_{-i}} \quad \Leftrightarrow \quad \tilde{z}_{-i}<\frac{\left(\nu_{1}+1\right)^{2}-\nu_{2}^{2}}{\kappa_{1}^{2}+\kappa_{2}^{2}+\xi_{1}^{2}+\xi_{2}^{2}+1}=\Psi_{1}
$$


Provided that other agents play the optimal $\omega$ given their suboptimal $\tilde{z}_{-i}$, the optimal strategy (11) is an equilibrium as long as the average attention of other players $\tilde{z}_{-i}$ is lower than the threshold value $\Psi_{1}$. By contrast, if the average attention of other players is higher than $\Psi_{1}$, it is no longer optimal for agent $i$ to play the optimal strategy (11). This means that when $\nu_{1}>\nu_{2}$ and $\Psi_{1}<\tilde{z}_{-i} \leq 1,\left\{\tilde{z}_{-i}=1, \omega\left(\tilde{z}_{-i}=1\right)\right\}$ is a suboptimal equilibrium.

Symmetrically, when $\nu_{1}<\nu_{2}$, the optimal strategy (12) with $z^{*}=1$ yields a higher utility to agent $i$ than the utility from the suboptimal strategy $\tilde{z}_{-i}$ when

$$
\left.\mathbb{E}\left(u_{i}\right)\right|_{z_{i}^{*}=1, \tilde{z}_{-i}}>\left.\mathbb{E}\left(u_{i}\right)\right|_{z_{i}=\tilde{z}_{-i}} \quad \Leftrightarrow \quad \tilde{z}_{-i}>\frac{\nu_{1}\left(\nu_{1}+1\right)-\nu_{2}\left(\nu_{2}+1\right)+\gamma\left(\kappa_{1}^{2}+\kappa_{2}^{2}\right)}{\kappa_{1}^{2}+\kappa_{2}^{2}+\xi_{1}^{2}+\xi_{2}^{2}+1}=\Psi_{2}(14
$$

The optimal strategy (12) is an equilibrium as long as the average attention of other players $\tilde{z}_{-i}$ is higher than the threshold value $\Psi_{2}$. By contrast, if the average attention of other players is lower than $\Psi_{2}$, it is no longer optimal for agent $i$ to play the optimal strategy (12). This means that when $\nu_{1}<\nu_{2}$ and $0 \leq \tilde{z}_{-i}<\Psi_{2},\left\{\tilde{z}_{-i}=0, \omega\left(\tilde{z}_{-i}=0\right)\right\}$ is a suboptimal equilibrium.

\subsection{Double overreaction in the optimal equilibrium}

In the beauty contest game with information acquisition, strategic complementarities give rise in the optimal equilibrium to a double overreaction to the signal with the highest common noise and lowest private noise. On the one hand, strategic complementarities increase the weight assigned to the most common and least private signal because it helps to coordinate better with other agents. This is the overreaction mechanism described in Morris and Shin (2002). On the other hand, strategic complementarities increase the attention given to the most common and least private signal, which in turn increases the weight assigned to that signal in the beauty contest game. Through their effect on information acquisition, strategic complementarities reinforce the overreaction to the most common and least private signal.

The overreaction due to the direct effect of strategic complementarities on the equilibrium weight in the action is obtained from varying $\gamma$ in (9) when the attention level $z$ is held constant. Differentiating $\omega$ with respect to $\gamma$ shows that increasing strategic complementarities increases the overreaction under the following conditions

$$
\frac{\partial \omega}{\partial \gamma} \lesseqgtr 0 \quad \Leftrightarrow \quad \frac{\kappa_{1}^{2}}{\xi_{1}^{2}+1-z} \lesseqgtr \frac{\kappa_{2}^{2}}{\xi_{2}^{2}+z}
$$

An increase in strategic complementarities increases the weight assigned to the signal with the largest ratio of common to private variances. Because common variance is less detrimental to utility when strategic complementarities are stronger, the most common and least private signal becomes more heavily weighted following an increase in strategic complementarities.

The second overreaction, in this case due to the indirect effect of strategic comple- 
mentarities on the equilibrium action through their effect on equilibrium attention, results from variations of $z$ in (9). The conditions for an increase in strategic complementarities to bring about a change in equilibrium attention $z$ are given in (11) and (12). An increase in $z$ from 0 to 1 yields an overreaction in $\omega$ of $1 /\left((1-\gamma)\left(\kappa_{1}^{2}+\kappa_{2}^{2}\right)+\xi_{1}^{2}+\xi_{2}^{2}+1\right)$.

Graphical illustration The double overreaction is illustrated in Figure 1. It plots the attention level $z$ (dashed lines) and weight $\omega$ (solid lines) assigned to signal $x_{1, i}$ when $\kappa_{1}^{2}=$ $2, \kappa_{2}^{2}=0.5$ and $\xi_{1}^{2}=0.5$ as a function of $\xi_{2}^{2}$ for two degrees of strategic complementarities, $\gamma=0$ (black) and $\gamma=0.85$ (blue).

When $\xi_{2}^{2}=0.375, \nu_{1}>\nu_{2}$, and optimal attention is $z^{*}=0$. Both signals have the same ratio of common to private variances $\kappa_{1}^{2} /\left(\xi_{1}^{2}+1\right)=\kappa_{2}^{2} / \xi_{2}^{2}$, which means that strategic complementarities do not lead to an overreaction to any of the two signals: with both $\gamma=0$ and $\gamma=0.85$, it is optimal to give zero attention and a weight of 0.2 to $x_{1, i}$ in the game.

When $\xi_{2}^{2}<0.375$, signal $x_{2, i}$ is more common and less private than signal $x_{1, i}$, so that strategic complementarities entail an overreaction to $x_{2, i}$ (i.e., an underreaction to $x_{1, i}$ ). This is illustrated by the difference - marked A in the figure - between $\left.\omega\right|_{\gamma=0.85}$ (solid blue line) and $\left.\omega\right|_{\gamma=0}$ (solid black line). This overreaction is due to the direct effect of strategic complementarities on optimal action. The attention level $z^{*}=0$ remains optimal for both values of $\gamma$, meaning that strategic complementarities do not affect agents' information acquisition.

When $\xi_{2}^{2}>0.375$, signal $x_{1, i}$ is more common and less private than signal $x_{2, i}$, so that strategic complementarities now entail an overreaction to $x_{1, i}$ (i.e., an underreaction to $x_{2, i}$ ). Strategic complementarities do not affect optimal attention when $0.375<\xi_{2}^{2}<$ 0.725 , in which case $z=0$ is optimal for both values of $\gamma$. Overreaction is illustrated by the difference - marked B in the figure - between $\left.\omega\right|_{\gamma=0.85}$ (solid blue line) and $\left.\omega\right|_{\gamma=0}$ (solid black line). Strategic complementarities do not affect optimal attention when $\xi_{2}^{2}>2$, in which case $z=1$ is optimal. Overreaction is illustrated by the difference - marked C in the figure - between $\left.\omega\right|_{\gamma=0.85}$ (solid blue line) and $\left.\omega\right|_{\gamma=0}$ (solid black line).

A double overreaction arises when strategic complementarities also affect optimal attention. This occurs when $0.725<\xi_{2}^{2}<2$ : optimal attention is $z^{*}=\left.0\right|_{\gamma=0}$ and $z^{*}=\left.1\right|_{\gamma=0.85}$. The double overreaction can be split between the effect of strategic complementarities on optimal action, on the one hand, and on optimal attention, on the other.

The dotted blue line shows $\omega$ when $\gamma=0.85$ but when the effect of strategic complementarities on optimal attention is ignored, i.e., while keeping $z=0$. The difference between the dotted blue line and the solid black line - marked D in the figure - captures the overreaction due to the effect of strategic complementarities on action without their effect on attention. The dotted black line shows $\omega$ when $\gamma=0$ but when the effect of strategic complementarities on optimal attention is ignored, i.e., while $z=1$. The difference between the solid blue line and the dotted black line - marked $\mathrm{E}$ in the figure captures the overreaction due to the effect of strategic complementarities on action without their effect on attention. Finally, the difference between the dotted black line and the 


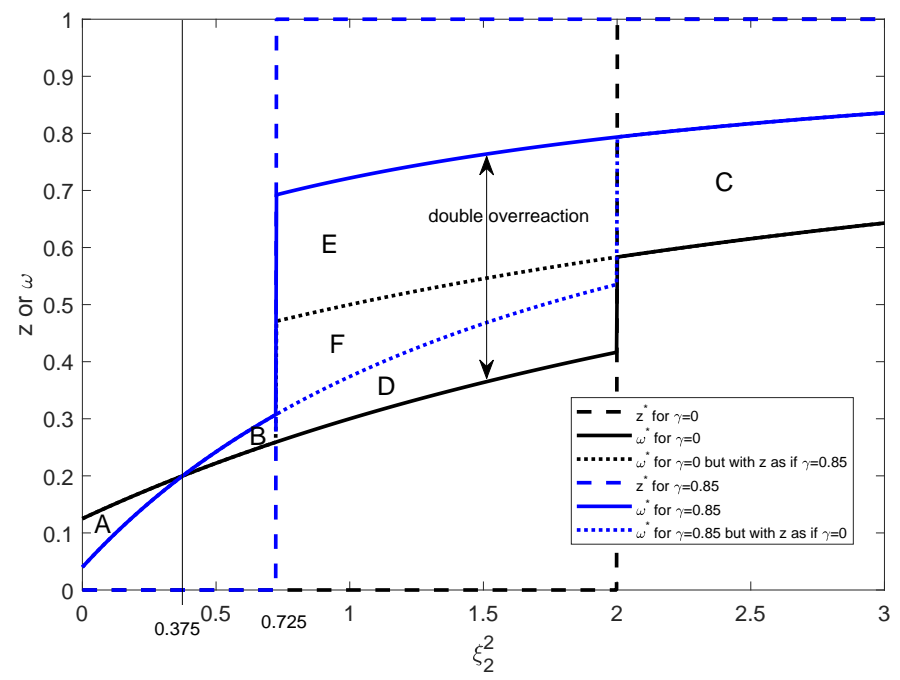

Figure 1: Decomposition of the double overreaction for $\kappa_{1}^{2}=2, \kappa_{2}^{2}=0.5$ and $\xi_{1}^{2}=0.5$ as a function of $\xi_{2}^{2}$

dotted blue line - marked $\mathrm{F}$ in the figure - shows the overreaction due exclusively to the effect of strategic complementarities on optimal attention.

\subsection{Theoretical predictions}

Our theoretical predictions focus on the optimal equilibrium because, as will become clear in sections 4 and 5 , this is the only relevant equilibrium given the parameter values in the experiment. The beauty contest game with information acquisition derived above provides several results that can be tested in the laboratory experiment. These results are summarised as follows:

Proposition 1 (attention) Full attention must be given to the signal with the lowest adjusted variance of error terms $(1-\gamma) \kappa^{2}+\xi^{2}$. An increase in strategic complementarities $\gamma$ tends to shift attention to the signal with the highest common noise and the lowest private noise.

Proposition 2 (double overreaction) Strategic complementarities give rise to a double overreaction to the signal with the highest common noise and the lowest private noise through (i) an increase in the signal's weight in equilibrium action over its weight in the expectation of the fundamental and (ii) an increase in equilibrium attention and in its weight in the expectation of the fundamental.

Proposition 3 (attention-action relation) The optimal weight assigned to a signal in the beauty contest increases with the attention given to the signal. Full attention is given to the signal with the greatest weight in the action. 


\subsection{Exogenous information}

In the experiment, to assess the role of information acquisition, the beauty contest game with information acquisition is compared to the beauty contest game with exogenous information. We thus present the theoretical counterpart under exogenous information. The variance of the common (sender) noise of signals $x_{1, i}$ and $x_{2, i}$ under exogenous information is identical to that under information acquisition. By contrast, the private (receiver) noise of signals $x_{1, i}$ and $x_{2, i}$ is given by $\epsilon_{1, i} \sim N\left(0, \sigma_{\epsilon, 1}^{2}\right)$ and $\epsilon_{2, i} \sim N\left(0, \sigma_{\epsilon, 2}^{2}\right)$ and independently distributed across agents. The variances of the private noises $\sigma_{\epsilon, 1}^{2}$ and $\sigma_{\epsilon, 2}^{2}$ are exogenous - that is, independent of the attention given to the signals.

The equilibrium action and expected utility are equivalent to those under endogenous information after we substitute the exogenous variance of idiosyncratic noises for the endogenous variance. The equilibrium action is determined by the weight assigned to the signal $x_{1, i}$

$$
\omega^{*}=\frac{(1-\gamma) \kappa_{2}^{2}+\sigma_{\epsilon, 2}^{2}}{(1-\gamma)\left(\kappa_{1}^{2}+\kappa_{2}^{2}\right)+\sigma_{\epsilon, 1}^{2}+\sigma_{\epsilon, 2}^{2}}
$$

and the unconditional expected utility is

$$
\mathbb{E}_{i}(u)=-\frac{\left((1-\gamma) \kappa_{1}^{2}+\sigma_{\epsilon, 1}^{2}\right)\left((1-\gamma) \kappa_{2}^{2}+\sigma_{\epsilon, 2}^{2}\right)}{(1-\gamma)\left(\kappa_{1}^{2}+\kappa_{2}^{2}\right)+\sigma_{\epsilon, 1}^{2}+\sigma_{\epsilon, 2}^{2}}
$$

\section{The experiment}

One may question whether the theoretical predictions above hold in practice when homines sapientes instead of homines oeconomici are involved in the beauty contest. A natural way to test this issue is to run a laboratory experiment that implements a beauty contest with information acquisition, as field data may be difficult to collect. The theoretical model in section 3 is perfectly suited to run an experiment. ${ }^{2}$ This section presents the experimental treatments and parameter values, the theoretically optimal behaviour, and the general procedure of the experiment.

\subsection{Treatments and parameters}

We run three experimental treatments. Treatment A consists of the beauty contest game with exogenous information. Treatment $\mathrm{B}$ consists of the beauty contest game with information acquisition. Treatment $\mathrm{C}$ consists of a fundamental-estimation game with information acquisition without strategic complementarities. In every treatment, the fundamental state $\theta$ is drawn randomly from a uniform distribution on the interval $[50,950] .{ }^{3}$ The parameter choices and equilibrium values are summarised in Table 1.

\footnotetext{
${ }^{2}$ The experiment involves a finite number of participants (instead of a continuum); however, we stipulate in the experiment that the average action is the action of others, implying that there is no theoretical difference between a model with a finite or an infinite number of agents.

${ }^{3}$ Participants were not told about the support of the distribution to avoid skewness in the posterior distribution.
} 
Treatment A Treatment A implements as a benchmark the beauty contest game with exogenous information as described in section 3.6. ${ }^{4}$ Each participant $i$ takes an action $a_{i}$ to maximise the payoff function in ECU (experimental currency units) given by the formula

$$
50-1.5\left(a_{i}-\theta\right)^{2}-8.5\left(a_{i}-\bar{a}_{-i}\right)^{2}
$$

where $\bar{a}_{-i}$ is the average action of other participants. Under exogenous information, each participant receives two signals on the fundamental $\theta$. The signal $x_{1}$ is common to all participants and contains an error term normally distributed with zero mean and variance $\kappa_{1}^{2}=2$. The signal $x_{2, i}$ is private to each participant and contains an idiosyncratic error term normally and independently distributed across participants with zero mean and variance $\sigma_{\epsilon, 2}^{2}=2$.

In addition to choosing their action $a_{i}$, participants are also required to reveal their expectations of the fundamental $\theta$ and of the decision of the other participants $\bar{a}_{-i}$. The elicitation of expectations is rewarded by $10 \mathrm{ECU}$ when the difference between the expected payoff based on these expectations and the realised payoff is not larger than $4 .^{5}$

Treatment B Treatment B implements the beauty contest game with information acquisition. The parameters are chosen to replicate treatment $\mathrm{A}$ in the optimal equilibrium. As in treatment $\mathrm{A}$, each participant $i$ takes an action $a_{i}$ to maximise the payoff function in ECU given by the formula

$$
50-1.5\left(a_{i}-\theta\right)^{2}-8.5\left(a_{i}-\bar{a}_{-i}\right)^{2}
$$

Under endogenous information, each participant allocates her attention of 1 between the two information sources $x_{1}$ and $x_{2}$. The signal $x_{1, i}$ contains an error term that is common to all participants and normally distributed with zero mean and variance $\kappa_{1}^{2}=2$ as well as an idiosyncratic error term that is private to each participant and normally and independently distributed across participants with zero mean and variance $1-z_{i}$ (i.e., $\xi_{1}^{2}=0$ ). The signal $x_{2, i}$ contains only an idiosyncratic error term that is private to each participant and normally and independently distributed across participants with zero mean and variance $1+z_{i}$ (i.e., $\xi_{2}^{2}=1$ ).

In addition to choosing their attention allocation $z_{i}$ and their action $a_{i}$, participants are also required to reveal their expectations of the fundamental $\theta$ and of the decisions of the other participants $\bar{a}_{-i}$. The elicitation of expectations is rewarded by 10 ECU when the difference between the expected payoff based on these expectations and the realised payoff is not larger than 4 .

\footnotetext{
${ }^{4}$ It thus replicates the beauty contest experiment with exogenous information by Baeriswyl and Cornand (2014) and Cornand and Heinemann (2014).

${ }^{5}$ Eliciting each participant's expectation about the fundamental and the average decision of other participants does not alter the incentive of the game because the reward is granted when the participant's elicited expectations are in line with her action. This ensures that the revealed expectations and the chosen action remain coherent.
} 


\begin{tabular}{|c|c|c|c|c|c|c|c||c|c|c|c|c|}
\hline Treatment & $\gamma$ & $\kappa_{1}^{2}$ & $\kappa_{2}^{2}$ & $\xi_{1}^{2}$ & $\xi_{2}^{2}$ & $\sigma_{\epsilon, 1}^{2}$ & $\sigma_{\epsilon, 2}^{2}$ & $z^{*}$ & $\omega^{*}$ & $u^{*}$ & $f$ & $o$ \\
\hline \hline $\mathrm{A}$ & 0.85 & 2 & 0 & - & - & 0 & 2 & - & 0.870 & -0.261 & 0.5 & 0.935 \\
\hline $\mathrm{B}$ & 0.85 & 2 & 0 & 0 & 1 & - & - & 1 & 0.870 & -0.261 & 0.5 & 0.935 \\
\hline $\mathrm{C}$ & 0 & 2 & 0 & 0 & 1 & - & - & 0 & 0.250 & -0.750 & 0.25 & - \\
\hline
\end{tabular}

Table 1: Experiment parameters and equilibrium

Treatment C Treatment $\mathrm{C}$ implements a fundamental-estimation game with information acquisition. Treatment $\mathrm{C}$ differs from treatment $\mathrm{B}$ only because the payoff function shows no strategic complementarities. The structure of information sources is the same as in treatment B. Each participant $i$ takes an action $a_{i}$ to maximise the payoff function in ECU given by the formula

$$
50-10\left(a_{i}-\theta\right)^{2}
$$

Although the expected fundamental should coincide with the action taken, participants are nevertheless required to reveal their expectations of the fundamental $\theta$, for the sake of symmetry with treatments A and B. The elicitation of expectations is rewarded as above.

\subsection{Testable implications}

The treatment and parameter choices allow us to test the following theoretical predictions in the experiment:

1. Full attention in treatment B: full attention $z^{*}=1$ to $x_{1, i}$ is the optimal equilibrium; zero attention $z=0$ is a suboptimal equilibrium, which yields a higher payoff to agent $i$ than choosing $z=1$ if the average attention of other agents is $\tilde{z}_{-i}<2.25 \%$, as expressed in (14).

2. Zero attention in treatment $\mathrm{C}$ : zero attention $z^{*}=0$ to $x_{1, i}$ is the optimal equilibrium; there is no suboptimal equilibrium, as expressed in (13).

3. Overreaction in treatments A and B: the optimal weight assigned to $x_{1, i}, \omega^{*}=0.87$, is identical in both treatments and is larger than the weight assigned without strategic complementarities $\left(\left.\omega\right|_{\gamma=0}=0.5\right.$ in $\mathrm{A}$ and $\left.\omega\right|_{\gamma=0}=0.25$ in B).

4. No overreaction in treatment C: the optimal weight assigned to $x_{1, i}, \omega^{*}=0.25$, is equal to the weight in the expectation of the fundamental.

5. Relationship between $z$ and $\omega$ in treatments B and C: in line with (8), attention and weight are strategic complements.

The upper graph in Figure 2 shows the optimal weight $\omega$ as a function of the attention level $z$ in treatment B (solid blue line, $\gamma=0.85$ ) and in treatment $\mathrm{C}$ (dashed black line, $\gamma=0$ ). The optimal weight $\omega$ increases linearly with the attention level $z$. The lower graph plots the corresponding utility. It also illustrates the fact that while multiple equilibria 

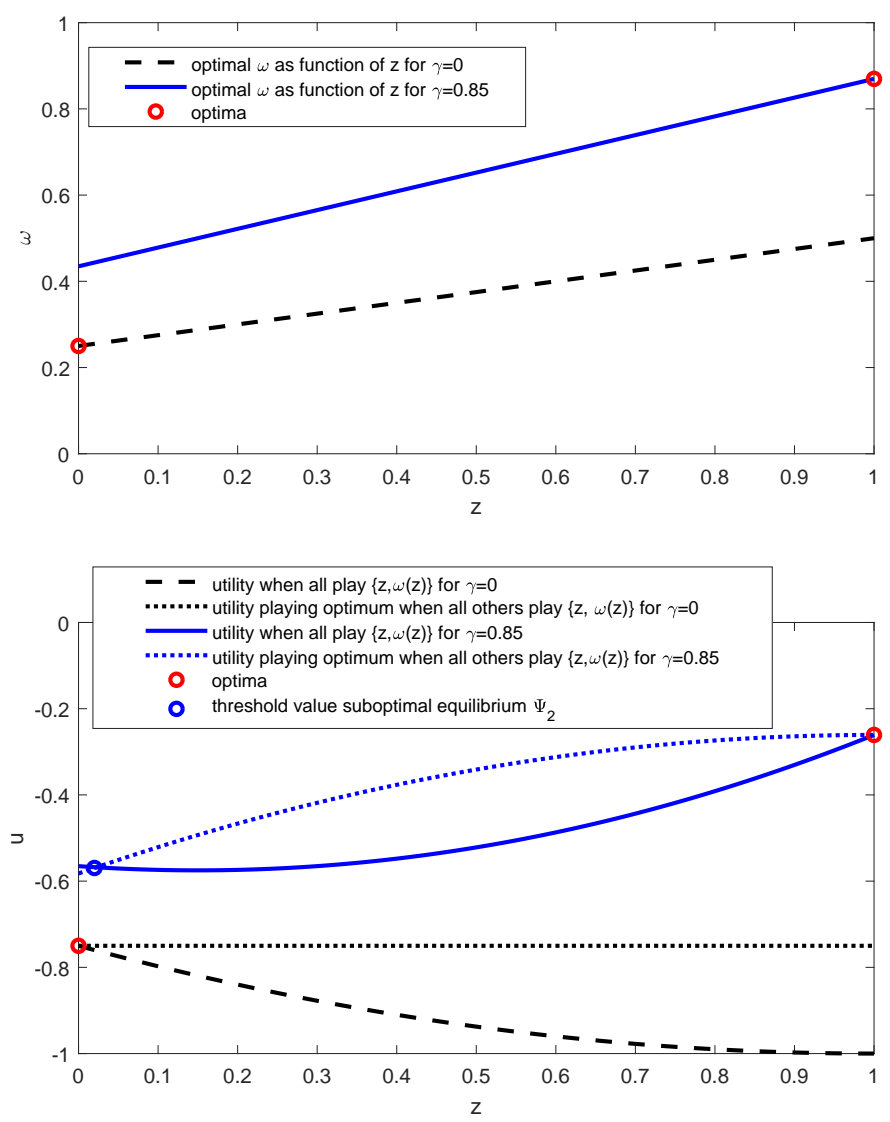

Figure 2: Optimal $\omega$ and utility as function of $z$ for $\kappa_{1}^{2}=2, \kappa_{2}^{2}=0, \xi_{1}^{2}=0$ and $\xi_{2}^{2}=1$

can occur with strategic complementarities (treatment B), the suboptimal equilibrium is very unlikely given the parameters chosen for the experiment. The blue solid line plots the expected utility of each agent when they all play $z$ and $\omega(z)$. The blue dotted line plots the expected utility of playing the optimal equilibrium $z^{*}=1$ and $\omega^{*}=0.870$ while all others play $z$ and $\omega(z)$. Playing the optimal equilibrium yields a superior payoff unless the average attention of all the others is below $2.25 \%$, which never occurs in the experiment. It is therefore always preferable to play $z=1$ independently of the chosen strategies of others.

Without strategic complementarities, the same exercise comparing the black line and the black dotted line shows that the optimal equilibrium is always unique.

\subsection{Procedure}

Each experimental session consisted of two games, and each game corresponded to a treatment. Each game was repeated for 15 periods (hence a total of 30 periods per session). Participants played within the same group during the whole length of the experiment and did not know the identity of the other participants in their group. In each session, the 18 participants were divided into three independent groups of 6 (to obtain 3 observations per session). 


\begin{tabular}{|c|c|c|c|c|}
\hline Session & Date & Groups & Periods 1-15 & Periods 16-30 \\
\hline \hline 1 & 19 December 2018 & $1-3$ & $\mathrm{~A}$ & $\mathrm{~B}$ \\
\hline 2 & 14 January 2019 & $4-6$ & $\mathrm{~A}$ & $\mathrm{~B}$ \\
\hline 3 & 22 January 2019 & $7-9$ & $\mathrm{~B}$ & $\mathrm{~A}$ \\
\hline 4 & 23 January 2019 & $10-12$ & $\mathrm{~B}$ & $\mathrm{~A}$ \\
\hline 5 & 10 January 2019 & $13-15$ & $\mathrm{C}$ & $\mathrm{B}$ \\
\hline 6 & 15 January 2019 & $16-18$ & $\mathrm{C}$ & $\mathrm{B}$ \\
\hline 7 & 22 January 2019 & $19-21$ & $\mathrm{~B}$ & $\mathrm{C}$ \\
\hline 8 & 23 January 2019 & $22-24$ & $\mathrm{~B}$ & $\mathrm{C}$ \\
\hline
\end{tabular}

Table 2: Experimental sessions and treatments

The sessions were run in December 2018 and January 2019 at the LEES (Laboratoire d'Economie Expérimentale de Strasbourg). ${ }^{6}$ Participants were mainly students from Strasbourg University (most were students in economics, mathematics, biology and psychology). Participants were seated in random order at PCs. Instructions were then read aloud, and questions were answered in private. Throughout the sessions, students were not allowed to communicate with one another and could not see each others screens. Each participant could only participate in one session. Before starting the experiment, participants were required to answer a few questions to ascertain their understanding of the instructions. Examples of the instructions and screens appear in the Appendix. After each period, participants were informed about the true fundamental, the other participants' decisions and their own payoffs. Information about past periods from the same game (including signals and their own decisions) was displayed during the decision phase on the lower part of the screen.

At the end of each session, 2 periods per game were selected to calculate payoffs. One hundred ECU converted to 12 euros. The payoffs ranged from 14 to 29 euros. The average payoff was approximately 24 euros. The sessions lasted for approximately 90 minutes. In all periods, it was not possible to earn negative payoffs.

\section{$5 \quad$ Experimental results}

The results of the laboratory experiment are presented according to the following structure. In section 5.1, we analyse the average behaviour across groups. In section 5.2, we break down the overreaction, overweighting and underweighting according to various possible sources of error. Section 5.3 compares participants' individual behaviour between treatments.

Statistical tests are based on Wilcoxon rank-sum tests when we compare the observed data to our theoretical predictions and on Wilcoxon matched-pairs signed-rank tests for the between-treatment tests.

\footnotetext{
${ }^{6}$ The project has been approved by the GATE-Lab local IRB.
} 


\subsection{Overall outcome and treatment comparison at the group level}

Table 3 reports the average attention level $z$ and the average weight $\omega$ realised in each group over all periods. Figure 3 plots the average attention level $z$ and the average weight $\omega$ realised in every period across all groups in treatments A, B and C. ${ }^{7}$ Figure 4 plots the distribution of attention $z$ and weight $\omega$ realised in each period by each participant.

\subsubsection{Measuring attention}

In accordance with theory, the attention given to signal $x_{1, i}$ is higher in $\mathrm{B}$ than in $\mathrm{C}$ $(p=0.001)$. Strategic complementarities have the expected effect on attention. However, attention is not as strong as theory predicts (i.e., $z<1$ ) in B and not as weak as theory predicts (i.e., $z>0)$ in $\mathrm{C}(p=0.000, p=0.000)$.

\begin{tabular}{|c||c|c||c|c|c|}
\hline \multicolumn{1}{|c||}{} & \multicolumn{2}{c||}{ Attention $z$} & \multicolumn{3}{c|}{ Weight $\omega$} \\
& B & C & A & B & C \\
\hline Group 1 & 0.777 & - & 0.776 & 0.708 & - \\
\hline Group 2 & 0.550 & - & 0.681 & 0.638 & - \\
\hline Group 3 & 0.776 & - & 0.617 & 0.546 & - \\
\hline Group 4 & 0.744 & - & 0.749 & 0.759 & - \\
\hline Group 5 & 0.646 & - & 0.588 & 0.637 & - \\
\hline Group 6 & 0.665 & - & 0.578 & 0.544 & - \\
\hline Group 7 & 0.918 & - & 0.708 & 0.657 & - \\
\hline Group 8 & 0.849 & - & 0.744 & 0.735 & - \\
\hline Group 9 & 0.766 & - & 0.840 & 0.810 & - \\
\hline Group 10 & 0.706 & - & 0.529 & 0.508 & - \\
\hline Group 11 & 0.773 & - & 0.650 & 0.673 & - \\
\hline Group 12 & 0.830 & - & 0.645 & 0.622 & - \\
\hline Group 13 & 0.702 & 0.361 & - & 0.562 & 0.379 \\
\hline Group 14 & 0.718 & 0.244 & - & 0.447 & 0.399 \\
\hline Group 15 & 0.589 & 0.388 & - & 0.525 & 0.311 \\
\hline Group 16 & 0.867 & 0.280 & - & 0.678 & 0.377 \\
\hline Group 17 & 0.695 & 0.329 & - & 0.612 & 0.402 \\
\hline Group 18 & 0.803 & 0.157 & - & 0.820 & 0.231 \\
\hline Group 19 & 0.672 & 0.336 & - & 0.579 & 0.476 \\
\hline Group 20 & 0.880 & 0.344 & - & 0.612 & 0.437 \\
\hline Group 21 & 0.864 & 0.436 & - & 0.622 & 0.416 \\
\hline Group 22 & 0.775 & 0.159 & - & 0.660 & 0.328 \\
\hline Group 23 & 0.903 & 0.442 & - & 0.666 & 0.328 \\
\hline Group 24 & 0.833 & 0.517 & - & 0.705 & 0.326 \\
\hline \hline Average & 0.763 & 0.333 & 0.675 & 0.639 & 0.368 \\
\hline \hline Equilibrium & 1 & 0 & 0.870 & 0.870 & 0.250 \\
\hline & & & & & \\
\hline
\end{tabular}

Table 3: Realised attention and weight

\footnotetext{
${ }^{7}$ The incentives to converge were not very strong around the equilibrium. The sensitivity of the payoffs to deviations from equilibrium attention $z$ and equilibrium weight $\omega$ is shown in Appendix A. This explains why we do not observe much learning. Figure 18 reported in Appendix B analyses convergence.
} 


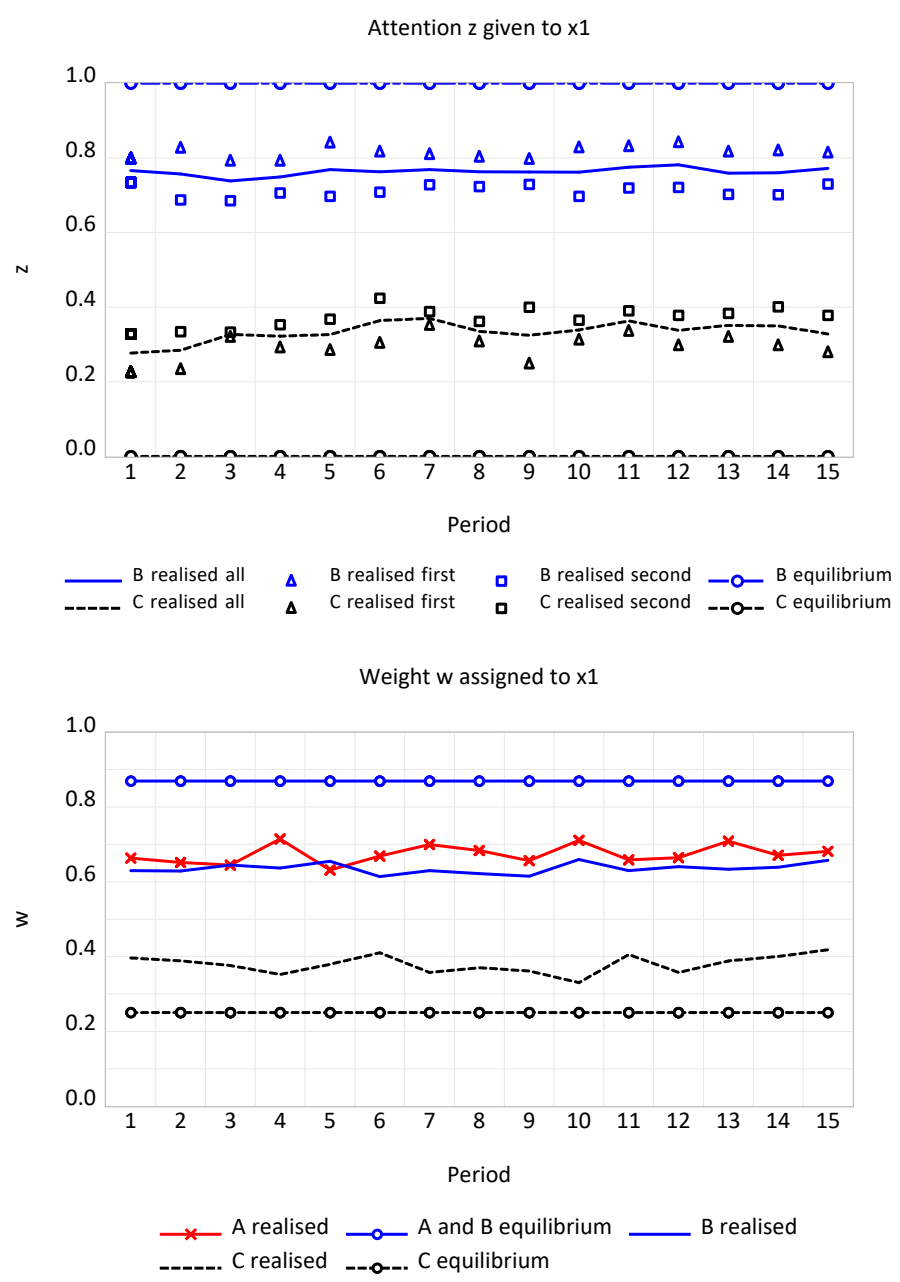

Figure 3: Averaged realised attention $z$ and weight $\omega$ given to $x_{1, i}$

The attention given to signal $x_{1, i}$ depends on the order of play of treatments. The triangles and squares in the upper panel of Figure 3 display the realised attention depending on whether the treatment in question comes first or second in the order of play. The blue (black) triangles display the realised attention when treatment $\mathrm{B}(\mathrm{C})$ is played first. The blue (black) squares display the realised attention when treatment B (C) is played second. The attention chosen in the first treatment tends to affect the attention chosen in the second treatment. Playing the low-attention treatment $\mathrm{C}$ first induces participants to choose a lower attention level in B than the level chosen when B is played first. Conversely, playing the high-attention treatment $\mathrm{B}$ first induces participants to choose a higher attention level in $\mathrm{C}$ than the level chosen when $\mathrm{C}$ is played first. This order effect does not, however, challenge the effect of strategic complementarities on attention: attention is always higher in $\mathrm{B}$ than in $\mathrm{C}$, independent of the order of play.

The analysis of realised attention shows that the suboptimal equilibrium derived in section 3.3.2 is never relevant in the experiment. As we outlined in section 4.2, the suboptimal equilibrium $z=0$ yields a higher payoff than the optimal equilibrium $z^{*}=1$ in $\mathrm{B}$ only if the others' average attention realised in B is below the threshold value of $2.25 \%$. 
Distribution of $w$ in treatment $A$
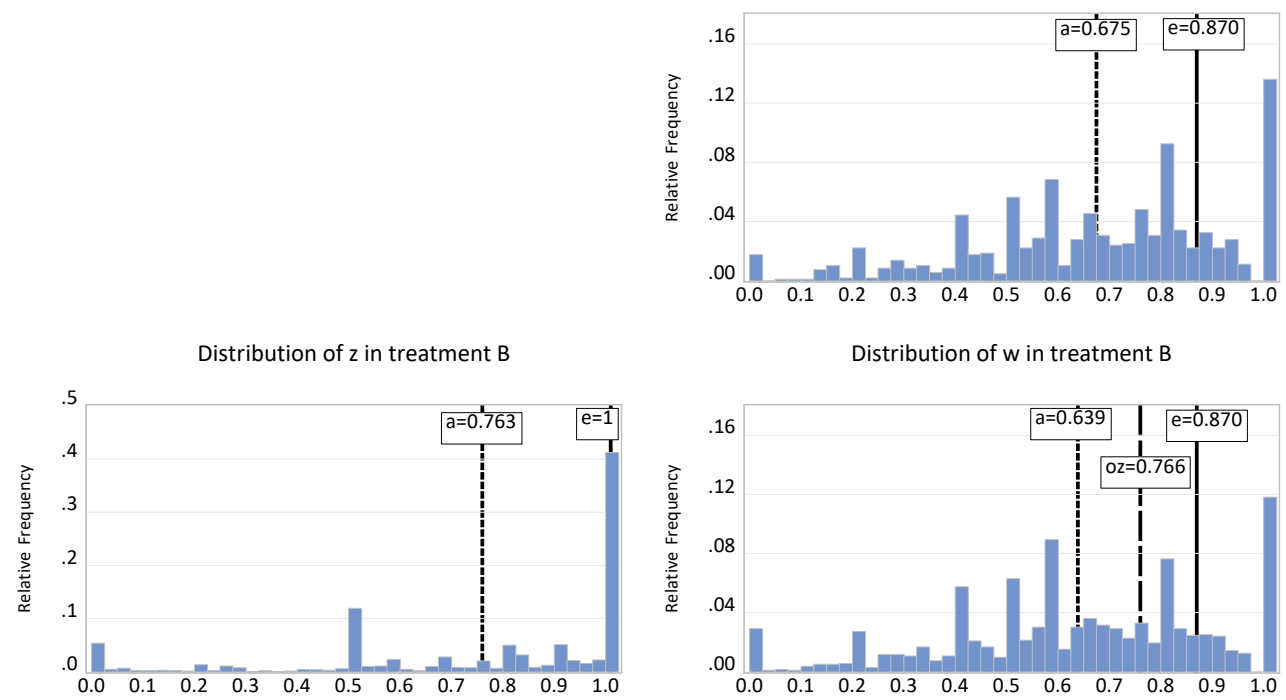

Distribution of $\mathrm{w}$ in treatment $\mathrm{B}$
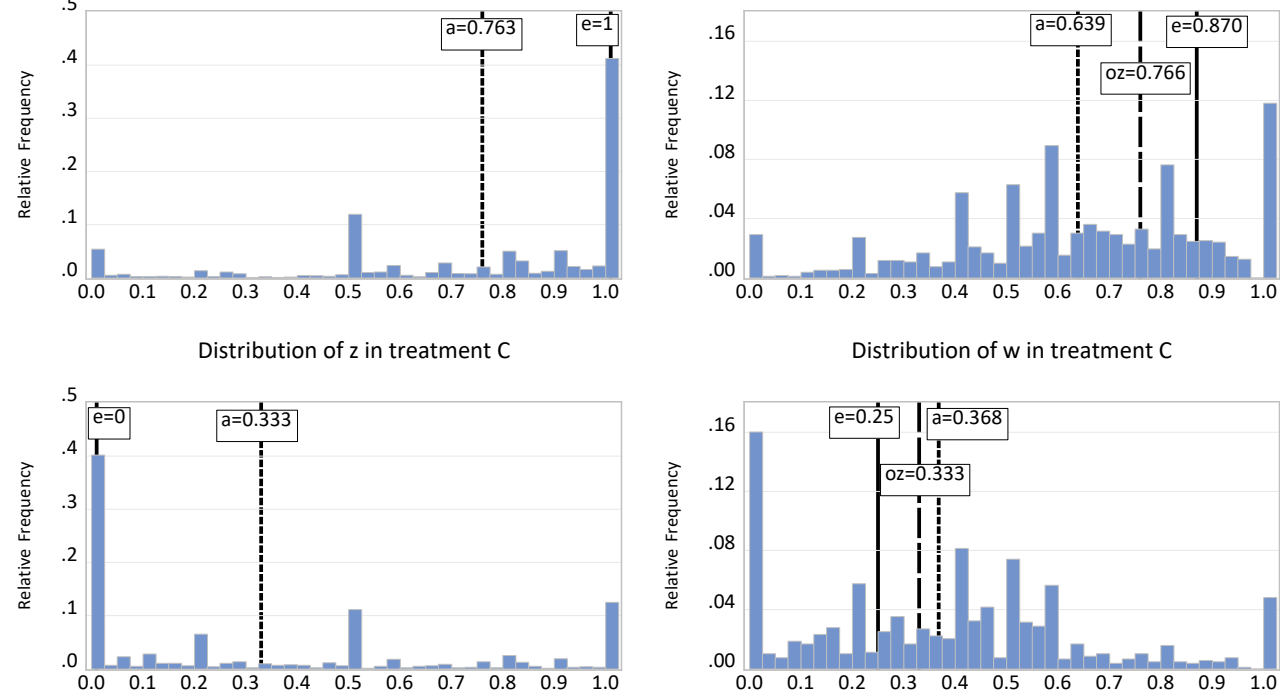

Distribution of $w$ in treatment $C$

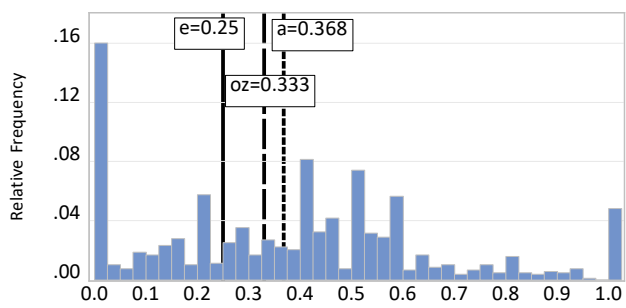

Figure 4: Distribution of attention $z$ and weight $\omega$; e: equilibrium; a: realised average; oz: average optimum given individually realised $z$.

Figure 19 in Appendix B shows, however, that the lowest average attention realised in treatment B within a group in any period is $45.8 \%$. This implies that it is never optimal for any participant in the experiment to choose $z=0$ at any period of treatment $\mathrm{B}$, making the suboptimal equilibrium irrelevant for our analysis.

Result 1 (a) Introducing strategic complementarities induces participants to pay more attention to $x_{1, i}$, in accordance with theory. Attention is, however, lower than theory predicts under strategic complementarities (treatment B) and higher than theory predicts without strategic complementarities (treatment $C$ ). (b) The suboptimal equilibrium under strategic complementarities (treatment B) is irrelevant in the experiment because the lowest average attention in any period is always higher than the threshold value at which the suboptimal equilibrium attention $z=0$ dominates the optimal equilibrium attention $z=1$. 


\subsubsection{Measuring overreaction}

In treatment $\mathrm{A}$, participants overreact to the public signal $x_{1}$ but not as much as theory predicts. ${ }^{8}$ Participants overreact to $x_{1}$ because the realised weight $\omega$ is significantly larger than the weight on $x_{1}$ without strategic complementarities, which corresponds to the weight on the expectation of the fundamental, $0.5(p=0.000)$. However, participants underweight $x_{1}$ because the realised weight $\omega$ is significantly smaller than the equilibrium weight in the beauty contest, $0.87(p=0.000)$. The average weight $\omega=0.66$ is close to the weight of 0.67 realised in a similar experiment by Baeriswyl and Cornand (2014).

In treatment $\mathrm{B}$, participants also overreact to the public signal $x_{1}$ but, again, not as much as theory predicts. The realised weight $\omega$ is significantly larger than the weight on $x_{1}$ without strategic complementarities, $0.25(p=0.000)$. The weight $\omega$ is significantly smaller than the equilibrium weight of $0.87(p=0.000)$. However, the weight $\omega$ is smaller than that in $\mathrm{A}$, although the equilibrium value of both weights is the same. The average weight amounts to 0.62 in $\mathrm{B}$, compared to 0.66 in $\mathrm{A}(p=0.084)$.

In treatment $\mathrm{C}$, participants overweight signal $x_{1, i}$. The realised weight $\omega$ is significantly greater than its equilibrium value of $0.25(p=0.000)$. In accordance with theory, the realised weight is significantly smaller than that in $\mathrm{B}(p=0.001)$.

Result 2 (a) Under strategic complementarities (treatments $A$ and $B$ ), participants overreact to $x_{1, i}$ as they assign a larger weight to it than in the treatment without strategic complementarities. (b) Under strategic complementarities (treatments $A$ and B), participants underweight $x_{1, i}$ as they assign a smaller weight to it than that predicted by theory. (c) The weight is lower with information acquisition (treatment B) than without information acquisition (treatment A). (d) Without strategic complementarities (treatment C), participants overweight $x_{1, i}$, as they assign a larger weight to it than that predicted by theory.

\subsection{Decomposing the overreaction, overweighting and underweighting}

The deviations of the realised weight $\omega$ from the optimal equilibrium can be broken down into four possible sources of error. The realised weight can deviate from equilibrium because

- participants pay suboptimal attention to $x_{1, i}$,

- participants form a suboptimal expectation of the fundamental given their information,

- participants form a suboptimal expectation of the average action of others given their information, or

\footnotetext{
${ }^{8}$ With regard to terminology, recall that 'overreaction' refers to the theoretical effect that strategic complementarities exert on the equilibrium weight $\omega$. By contrast, we use the terms 'overweighting' and 'underweighting' to refer to deviations from equilibrium in the weight realised in the experiment.
} 

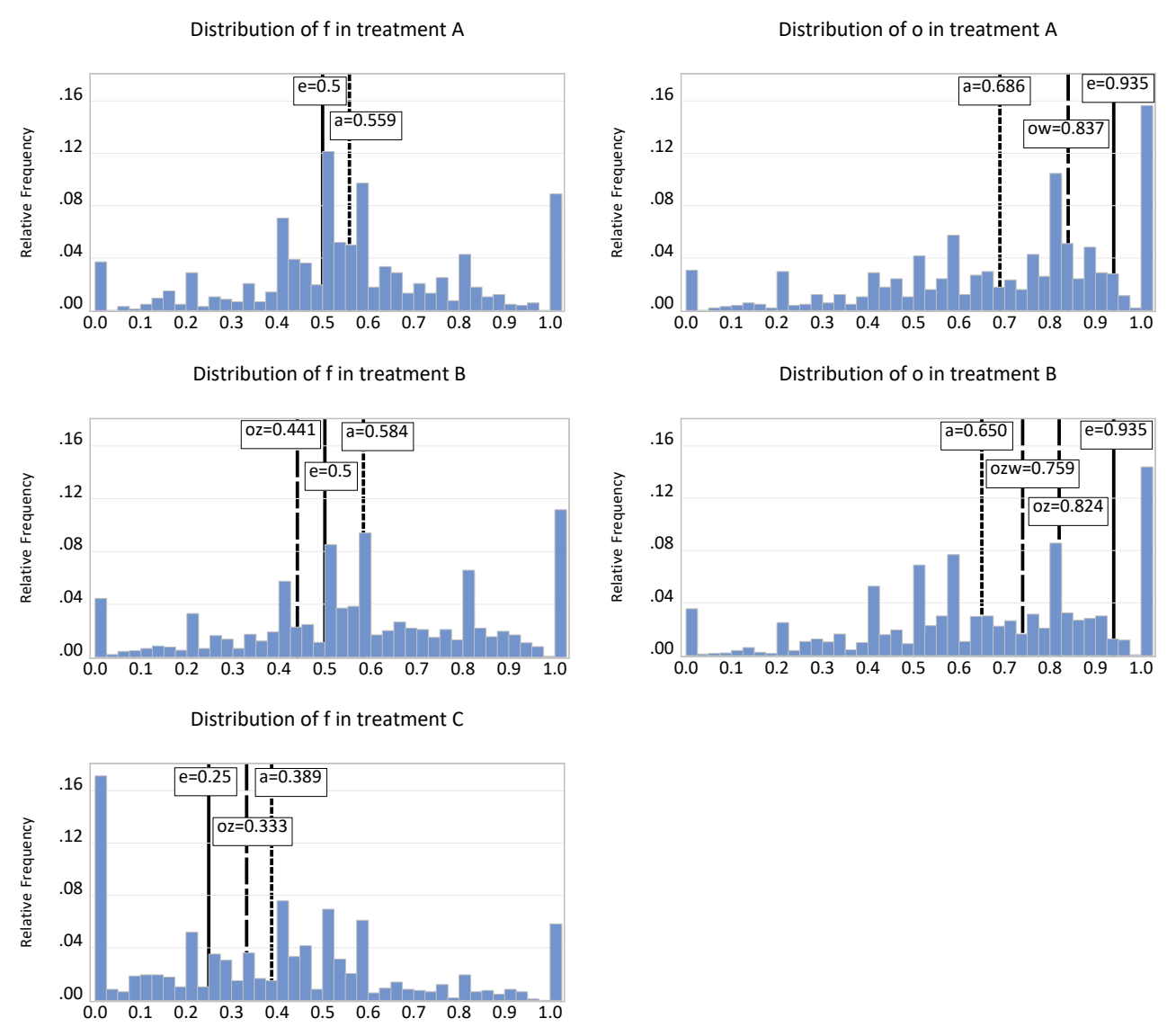

Figure 5: Distribution of weights assigned to $x_{1, i}$ in the expectations of fundamental $\theta$ and of others' action $\bar{a}_{-i}$; e: equilibrium; a: realised average; oz: average optimum given individually realised $z$; ozw: average optimum given individually realised $z$ and $\omega$.

- participants choose a suboptimal weight on $x_{1, i}$ in their action given their expectation about the fundamental and the action of others.

Figure 5 plots the distribution of realised weight assigned to $x_{1, i}$ in the expectation of the fundamental $f$ and in the expectation of others' action $o$ for treatments $\mathrm{A}, \mathrm{B}$ and $\mathrm{C}$; the theoretical values of these variables are derived in section 3.3.1.

\subsubsection{Treatment A}

Figure 4 (upper right panel) plots the relative frequency of weights $\omega$ in treatment A. The distribution exhibits a focal point in $\omega=1$. The realised weights are mainly concentrated between 0.5 and 1 . As already emphasised, the realised average $\omega$ in treatment $\mathrm{A}$ is significantly lower than its equilibrium value. Figure 6 breaks down the deviations of the realised $\omega$ from its equilibrium by the four types of error.

Error in attention In treatment A, because information is exogenous, there is no attention to allocate, so suboptimal attention cannot be the cause of a suboptimal equilibrium. Signal $x_{1, i}$ is always common knowledge, while signal $x_{2, i}$ is fully private. 

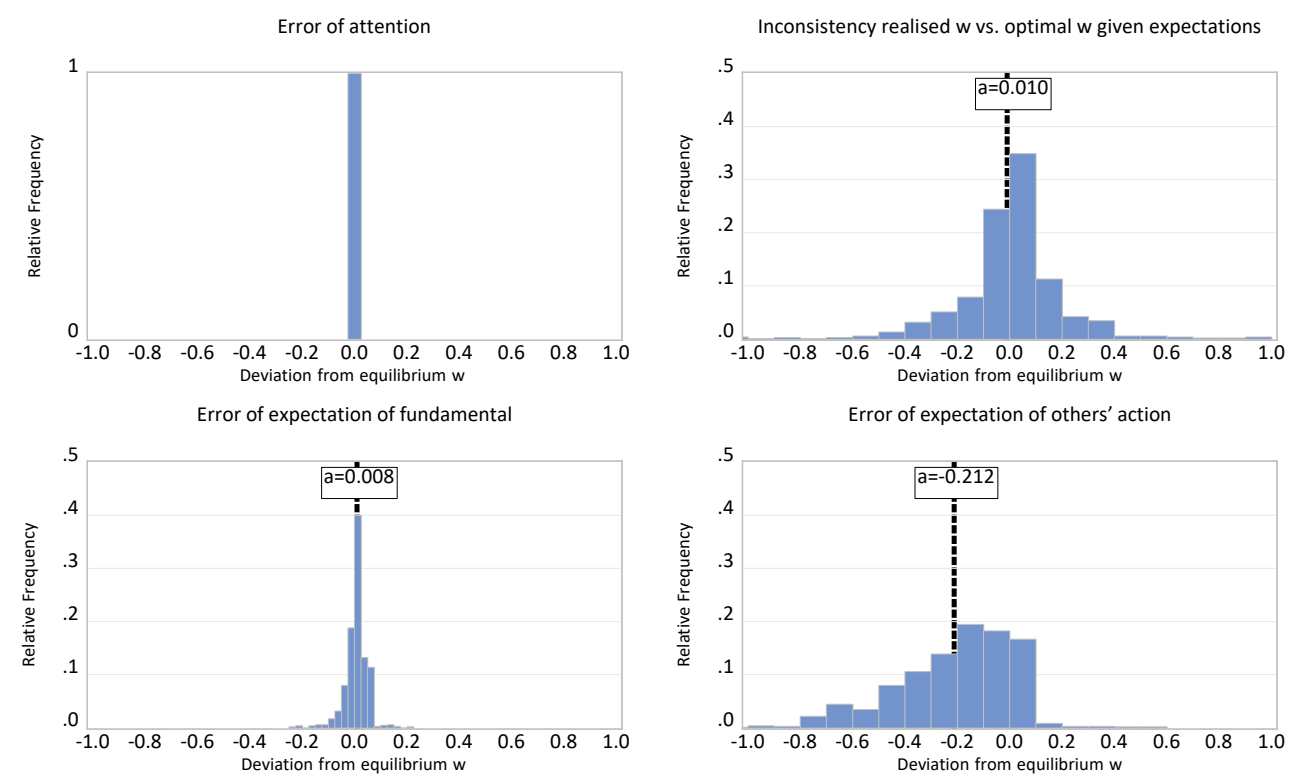

Figure 6: Decomposition of deviation of realised $\omega$ from equilibrium by error types in treatment A

Error in the expectation of the fundamental Although expectations of the fundamental $\theta$ are biased upward, they contribute very little to the suboptimal realised weight $\omega$. Figure 5 (upper left panel) plots the relative frequency of $f$, the weight assigned to $x_{1, i}$ in the expectation of the fundamental $\theta$. Errors in the expectation of the fundamental only contribute to raising the beauty contest weight by $0.8 \mathrm{pp}$ from its optimal equilibrium because the contribution of the quadratic distance to the fundamental is small in the utility function (1.5) in comparison to the quadratic distance to the average action of others (8.5).

The excessively large weight in the expectation of the fundamental can be explained by the positive correlation observed between the weight in the fundamental expectation and the weight chosen in the action. Participants tend to weight $x_{1, i}$ higher in the expectation of the fundamental $f$ when they weight it higher in the beauty contest action $\omega$, although in theory $f$ is independent of $\omega$ (see Figure 20 (a) in Appendix B). That $\omega>0.5$ on average can explain why the realised $f$ is larger than is optimal.

Error in the expectation of the actions of others The error in the expectation of the action of others contributes the largest part of the deviation of the realised $\omega$ from its optimal equilibrium. The upper right panel of Figure 5 plots the relative frequency of the weight assigned to $x_{1, i}$ in the expectation of the action of others for treatment $\mathrm{A}$. Because the contribution of the quadratic distance to the action of others is large (8.5) in the utility function, errors in this expectation contribute to reducing the beauty contest weight by $21.2 \mathrm{pp}$ from its optimal equilibrium.

The realised average weight $o$ is below the equilibrium value (although $\omega=1$ is focal) and below the weight conditional on participants' individually realised weight (ow), mean- 
ing that the deviation of $o$ from equilibrium cannot only be explained by the individually realised weight $\omega$. As theory predicts, participants tend to weight $x_{1, i}$ higher in their expectation of the action of others $o$ when they weight it higher themselves in action $\omega$. The positive realised relationship between $\omega$ and $o$ and the positive theoretical relationship between both are depicted in Figure 20 (b) in Appendix B.

Inconsistency between expectations and action The weight $\omega$ in the action is not systematically inconsistent with participants' expectation of the fundamental and of the actions of others. Figure 6 (upper right panel) shows the inconsistency between the elicited expectations $f$ and $o$, and the chosen action $\omega$ only accounts for $0.8 \mathrm{pp}$ of the deviation in treatment $\mathrm{A}$.

Result 3 (a) Without information acquisition (treatment A), the public signal plays a focal role. (b) The excessively low weight realised in the beauty contest $\omega$ can be explained by the excessively low expectation of the actions of others.

\subsubsection{Treatment B}

Figure 4 (middle panels) presents the relative frequency of realised attention $z$ and realised weight $\omega$ on $x_{1, i}$ in the beauty contest action in treatment $\mathrm{B}$. As in treatment $\mathrm{A}$, the distribution exhibits a focal point in $\omega=1$, and the realised average weight is significantly lower than its equilibrium value. Figure 7 illustrates the realised $z$ and $\omega$ in B for each of the 1080 individual decisions (12 groups x 6 participants x 15 periods). As theory predicts in (8), participants tend to play a higher weight $\omega$ when they have chosen a higher attention level $z$. The realised relationship (solid blue line) is nevertheless weaker than the theoretical one (dashed black line). Figure 8 breaks down the deviation of the realised $\omega$ from its equilibrium by error type.

Error in attention A large part of the negative deviation of the realised $\omega$ from equilibrium can be explained by an excessively low realised attention level $z$. The middle left panel in Figure 4 shows that more than $40 \%$ of subjects play the equilibrium attention $z=1$. More than $10 \%$ play the mid-point $z=0.5$, and approximately $5 \%$ paid no attention $z=0$ to $x_{1, i}$. The other realised $z$ levels are mainly distributed between 0.5 and 1. The average realised attention level is lower in B than theory predicts, meaning that $x_{1, i}$ contains private noise. Figure 8 (upper left panel) shows that errors in attention contribute to reducing the beauty contest weight by $10.2 \mathrm{pp}$ from its optimal equilibrium.

Figure 9 provides an alternative illustration of the effect of the realised attention level $z$ on the average optimal weight $\omega$ per period. Given the realised attention level $z$, the optimal weights assigned to $x_{1, i}$ in the beauty contest (blue stars) as well as in the expectation of the fundamental (red stars) are significantly below their equilibrium values (blue and red circles). 


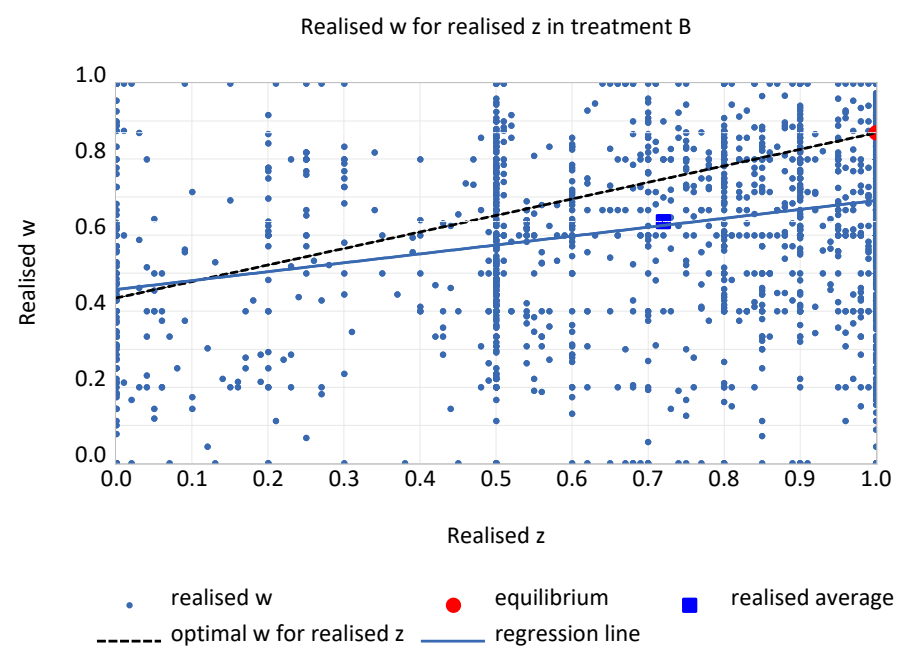

Figure 7: Realised $\omega$ for realised $z$ in treatment B
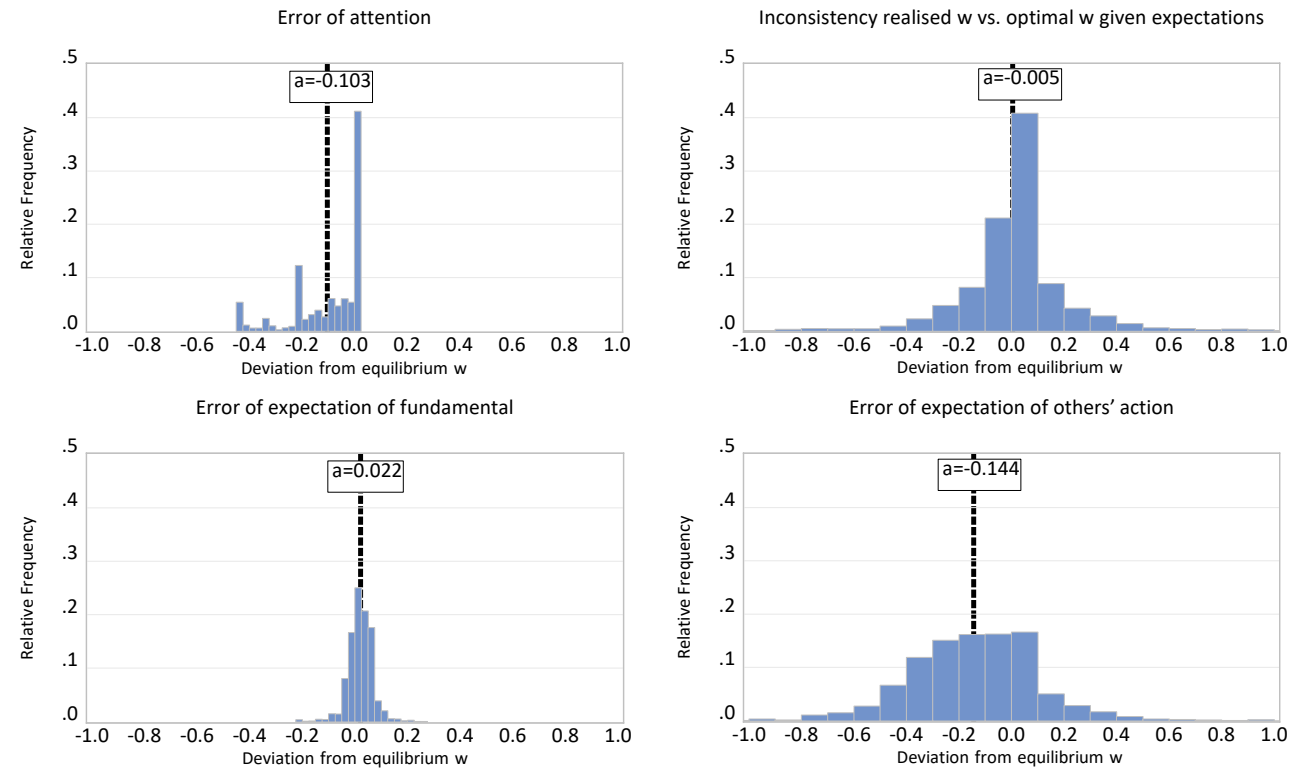

Figure 8: Decomposition of deviation of realised $\omega$ from equilibrium by error types in treatment B

Error in the expectation of the fundamental As in treatment A, errors in the expectation of the fundamental do not account for much of the suboptimal weight on $x_{1, i}$ in treatment B. The middle left panel of Figure 5 plots the relative frequency of weight $f$ assigned to $x_{1, i}$ in the expectation of the fundamental $\theta$. The realised average weight $f=0.58$ is larger than its equilibrium value $(0.5)$. However, as the realised attention level $z$ is lower than equilibrium attention, it would have been optimal to choose a lower $f=0.44$. Because the contribution of the quadratic distance from the fundamental is small in the utility function (1.5) in comparison to the quadratic distance to the average actions of others (8.5), errors in the expectation of the fundamental only contribute to 


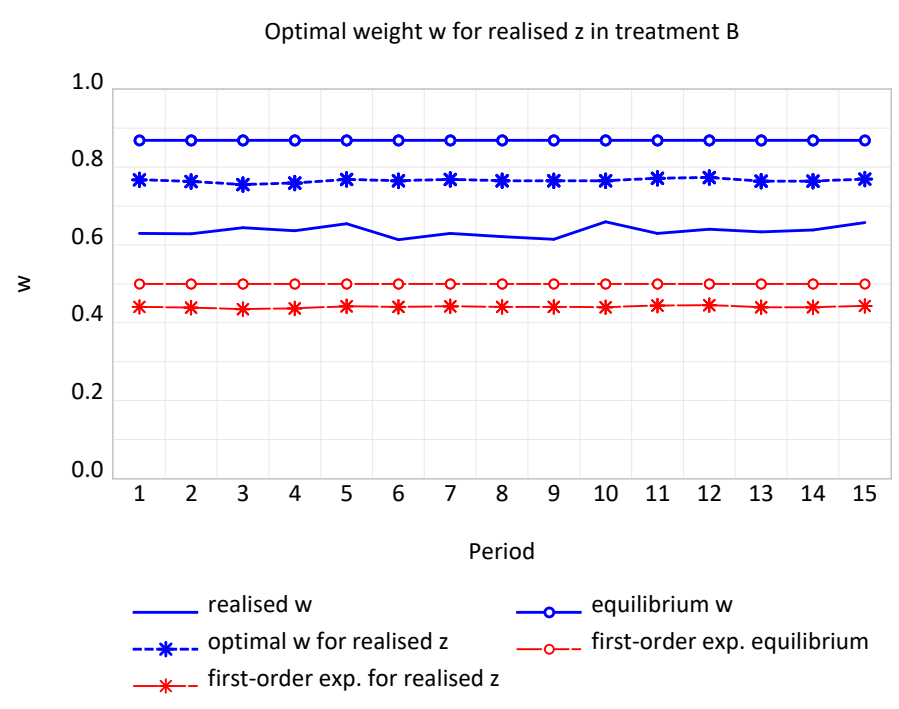

Figure 9: Optimal $\omega$ for realised $z$ in treatment B

raising the beauty contest weight by $0.5 \mathrm{pp}$ from its optimal equilibrium.

The realised weight $f$ increases with the realised attention level $z$ as theory predicts, although the realised $f$ is systematically higher than its theoretical level (see Figure 21 (a) in Appendix B). However, contrary to theoretical predictions, the realised weight $f$ also increases with the part of the realised $\omega$ that is not due to realised attention, that is, with the difference between the realised $\omega$ and the optimal $\omega$ given the realised $z$; this difference captures the variations in realised $\omega$ that cannot be explained by realised $z$ (see Figure 21 (b) in Appendix B).

Error in the expectation of the actions of others A large part of the deviation of the realised $\omega$ from its optimal equilibrium can be explained by biased expectations of the actions of others. The middle right panel of Figure 5 plots the relative frequency of weight assigned to $x_{1, i}$ in the expectation of the actions of others. The lower right panel of Figure 8 points out that errors in forming expectations about other participants' actions contribute to reducing the beauty contest weight by $14.4 \mathrm{pp}$ from its optimal equilibrium.

The realised average weight $o$ is below the equilibrium value (although $\omega=1$ is focal) and below the weight conditional on participants' individually realised attention $(o z)$ or conditional on both participants' individually realised attention and the weight (ozw). Participants tend to weight $x_{1, i}$ in the expectation of others' action more when they attribute more attention to it, as theory predicts, although the realised $o$ is systematically lower that its theoretical level (see Figure 22 (a) in Appendix B). The realised weight $o$ also increases with the difference between the realised $\omega$ and the optimal $\omega$ given the realised attention level $z$ (see Figure 22 (b) in Appendix B), which indicates that, in line with theoretical predictions, the realised $o$ tends to increase with deviations of $\omega$ that are not rationalised by the choice of attention level $z$. 


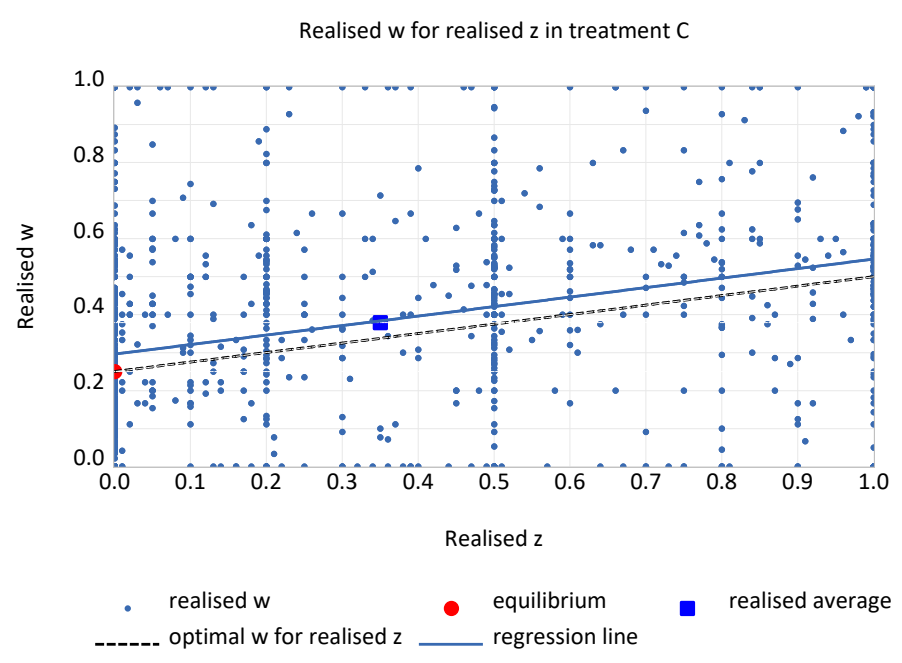

Figure 10: Realised $\omega$ for realised $z$ in treatment $\mathrm{C}$

Inconsistency between expectations and action The weight $\omega$ in the action is not systematically inconsistent with participants' expectations of the fundamental and of the actions of others. Figure 8 (upper right panel) shows that the inconsistency between the elicited expectations $f$ and $o$ and the chosen action $\omega$ only account for $0.5 \mathrm{pp}$ of the deviation.

Result 4 (a) Under strategic complementarities and with information acquisition (treatment B), participants increase the weight $\omega$ with the attention level $z$, but not as much as theory predicts. (b) The excessively low weight realised in the beauty contest $\omega$ can be explained by the excessively low realised attention level $z$ and the excessively low expectation about the actions of others $o$.

\subsubsection{Treatment $\mathrm{C}$}

Figure 4 (lower panels) presents the relative frequency of the realised attention level $z$ and weight $\omega$ on $x_{1, i}$ in the fundamental-estimation game in treatment $\mathrm{C}$. The distribution exhibits a focal point at $\omega=0$, and the realised average weight is significantly higher than its equilibrium value. Figure 10 illustrates the realised $z$ and $\omega$ values for each of the 540 individual decisions (6 groups x 6 participants $\times 15$ periods). The solid blue line shows that participants tend to increase $\omega$ with $z$, as theory predicts in (8). This line is slightly higher than the dashed black line depicting the optimal weight $\omega$ for the realised attention level $z$. This means that for their realised $z$ values, participants play a weight $\omega$ that is slightly larger than optimal. Figure 11 presents the decomposition of the deviation from the equilibrium weight $\omega$ by error type in treatment $\mathrm{C}$.

Error in attention A large part of the positive deviation of the realised $\omega$ from its equilibrium can be explained by an excessively high realised attention level $z$. The lower 

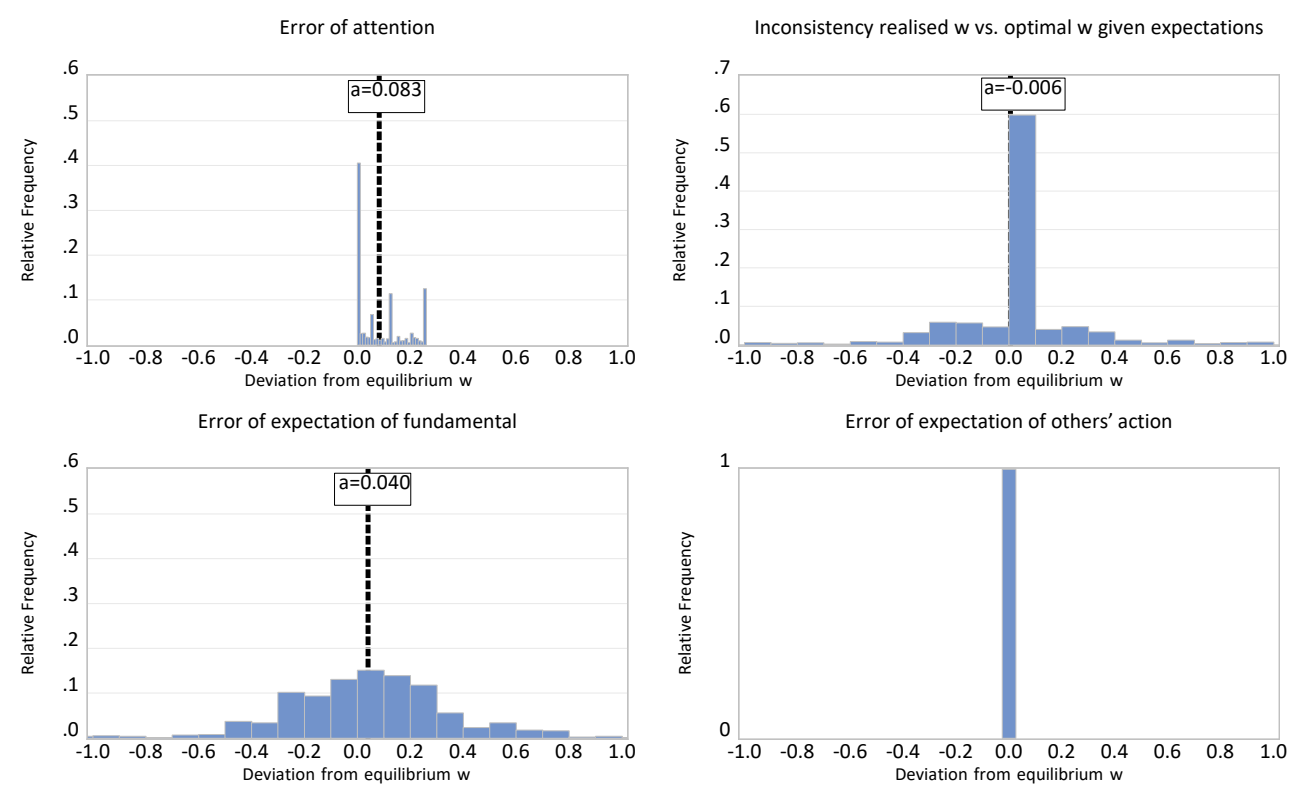

Figure 11: Decomposition of deviation of realised $\omega$ from equilibrium by error types in treatment $\mathrm{C}$

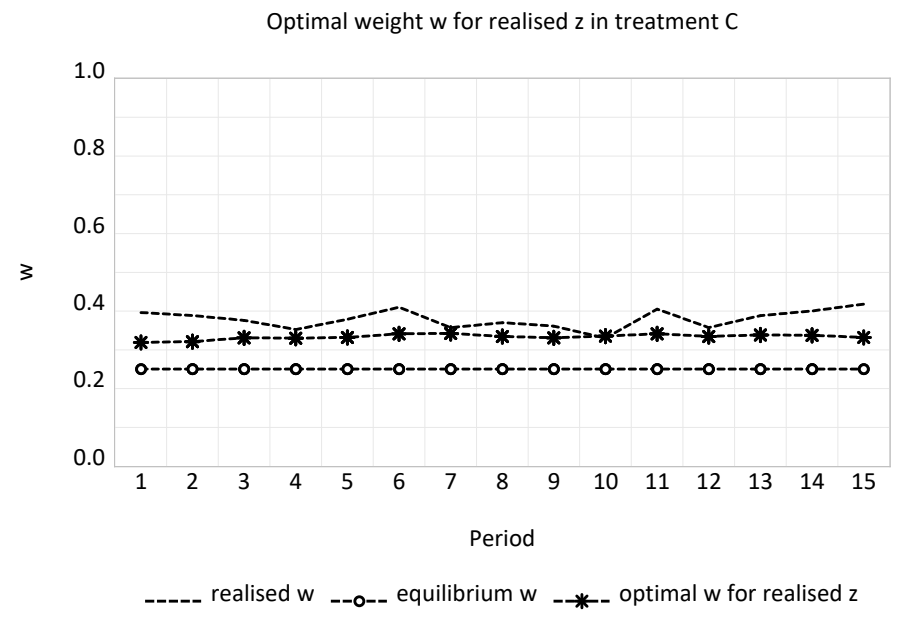

Figure 12: Optimal $\omega$ for realised $z$ in treatment $\mathrm{C}$

left panel of Figure 4 shows that while approximately $40 \%$ of participants attribute no attention to signal $x_{1, i}$, in line with theoretical predictions, it seems that $z=0.5$ and $z=1$ are focal since more than $20 \%$ of subjects play one strategy or the other. Figure 11 (upper left panel) shows that errors in attention contribute to increasing the weight $\omega$ by $8.3 \mathrm{pp}$ above its optimal equilibrium.

Figure 12 provides an alternative illustration of the effect of realised attention $z$ on the average optimal weight $\omega$ per period. Given the realised attention values, the optimal weight assigned to $x_{1, i}$ in the beauty contest (black stars) is significantly above its equilibrium value (black circles). 
Error in the expectation of the fundamental Part of the positive deviation of the realised $\omega$ from its equilibrium can also be explained by errors in the expectation of the fundamental. The lower panel of Figure 5 plots the relative frequency of the weight assigned to $x_{1, i}$ in the expectation of the fundamental. The realised average weight $f=$ 0.39 is larger than its equilibrium value $(0.25)$ but in the vicinity of the realised average weight in action $\omega=0.38$. As the realised attention level $z$ is larger than equilibrium attention, it would have been optimal to choose on average $f=0.33$, which is still smaller than the realised $f$ but larger than the equilibrium $f$. In contrast to the utility functions of treatments $\mathrm{A}$ and $\mathrm{B}$, utility in treatment $\mathrm{C}$ depends only on the quadratic distance to the fundamental, implying that errors in forming fundamental expectations matter more than in A and B. Errors in the expectation of the fundamental contribute to raising the weight $\omega$ by 4 pp from its optimal equilibrium.

The realised weight $f$ increases with the realised attention level $z$, as theory predicts (see Figure 23 (a) in Appendix B). However, contrary to theoretical predictions, the realised weight $f$ also increases with the difference between the realised $\omega$ and the optimal $\omega$ given the realised $z$, which captures the variations in realised $\omega$ that cannot be explained by the choice of $z$ (see Figure 23 (b) in Appendix B).

Error in the expectation of the actions of others The expectation of the actions of others is not elicited in treatment $\mathrm{C}$ because this expectation is irrelevant without strategic complementarities.

Inconsistency between expectations and action The weight $\omega$ in the action is not systematically inconsistent with participants' expectation of the fundamental. Figure 11 (upper right panel) shows that the inconsistency between the elicited expectations $f$ and the chosen action $\omega$ only accounts for $0.6 \mathrm{pp}$ of the deviation.

Result 5 (a) Without strategic complementarities (treatment C), participants increase the weight $\omega$ with the attention level $z$ by slightly more than what theory predicts. (b) The excessively high realised weight $\omega$ in the fundamentalestimation game can be explained by the excessively high realised attention level $z$ and the excessively high expectation of the fundamental $f$.

\subsubsection{Decomposing the double overreaction}

As discussed in section 3.4, strategic complementarities give rise to a double overreaction to $x_{1, i}$. Combining the results of sections 5.2.2 and 5.2.3 allows us to decompose the double overreaction into the effect of strategic complementarities on the realised attention level $z$ and on the realised weight $\omega$, as plotted in Figure 13.

In theory, increasing $\gamma$ from 0 to 0.85 increases the equilibrium attention level $z$ from 0 to 1 and the weight on $x_{1, i}$ in the expectation of the fundamental $\theta$ from 0.25 to 0.5 . Increasing $\gamma$ from 0 to 0.85 also increases the equilibrium weight in the beauty contest $\omega$ to 


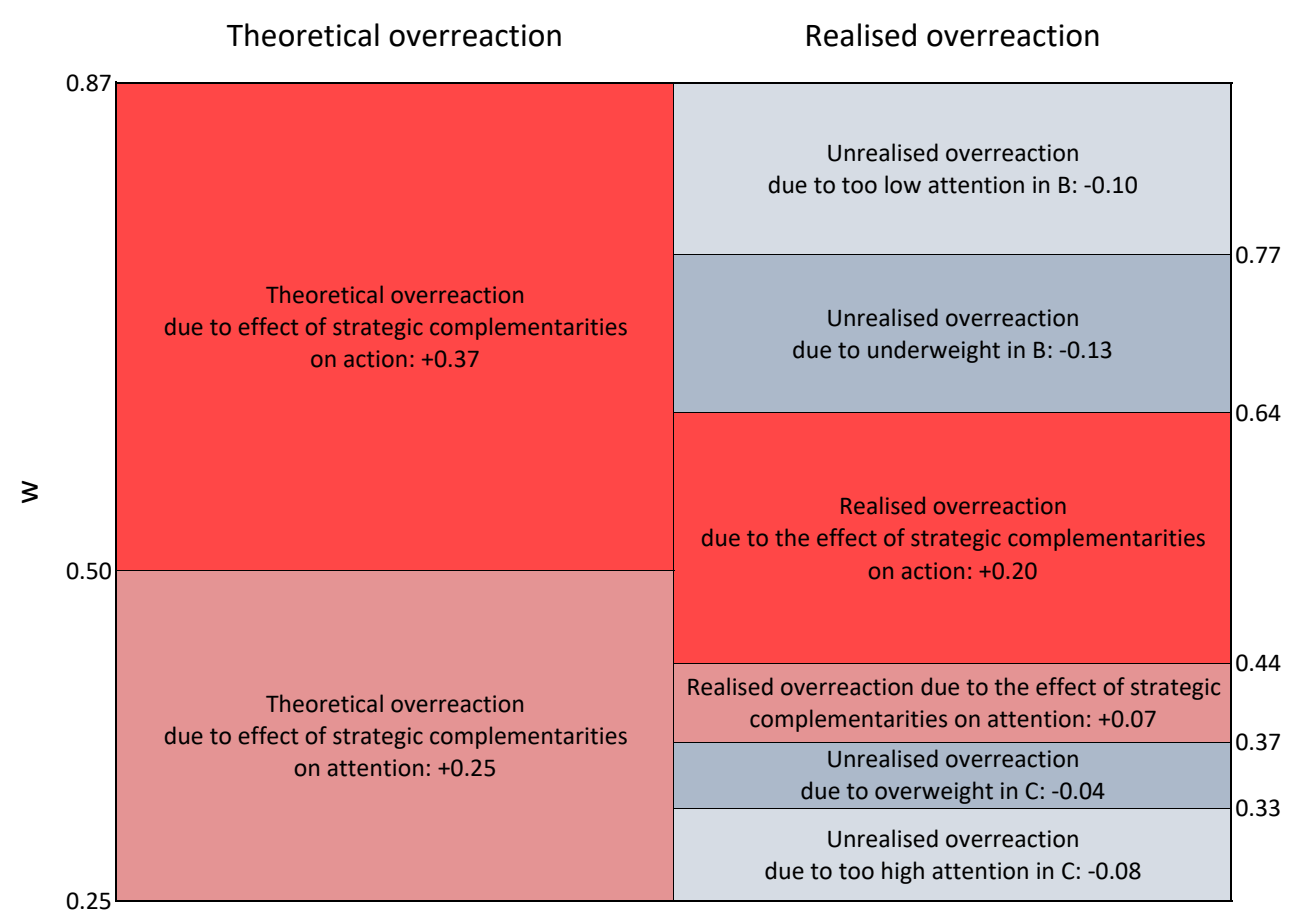

Figure 13: Theoretical and realised decomposition of double overreaction into the effect of strategic complementarities on realised attention $z$ and on realised weight $\omega$

0.87. When both effects are combined, introducing strategic complementarities increases the equilibrium weight assigned to $x_{1, i}$ from 0.25 to 0.87 .

In the experiment, the effect of strategic complementarities is much smaller than that in theory: the average realised weight of 0.37 in $\mathrm{C}$ rises to 0.64 in $\mathrm{B}$. The realised overreaction amounts to approximately $44 \%$ of the theoretical prediction. Approximately half of the unrealised overreaction is caused by the suboptimal allocation of attention (too high in $\mathrm{C}$, too low in B) and the other half by the suboptimal action given the realised attention level (overweighting in $\mathrm{C}$, underweighting in $\mathrm{B}$ ).

Result 6 (a) The realised double overreaction amounts to approximately $44 \%$ of the theoretical prediction. (b) The unrealised double overreaction is caused equally by the suboptimal allocation of attention and by the suboptimal action given the realised attention level.

\subsection{Comparison of participants' behaviour between treatments}

This section compares participants' behaviour between treatments. It examines whether the behavioural pattern in one treatment predicts the behavioural pattern in another treatment.

The upper panel of Figure 14 plots the average error of each participant in the realised weight $\omega$ in $B$ and in $A$. The average error in the realised weight $\omega$ in $B$ is conditional on the realised individual attention level in each period. The positive regression line indicates 

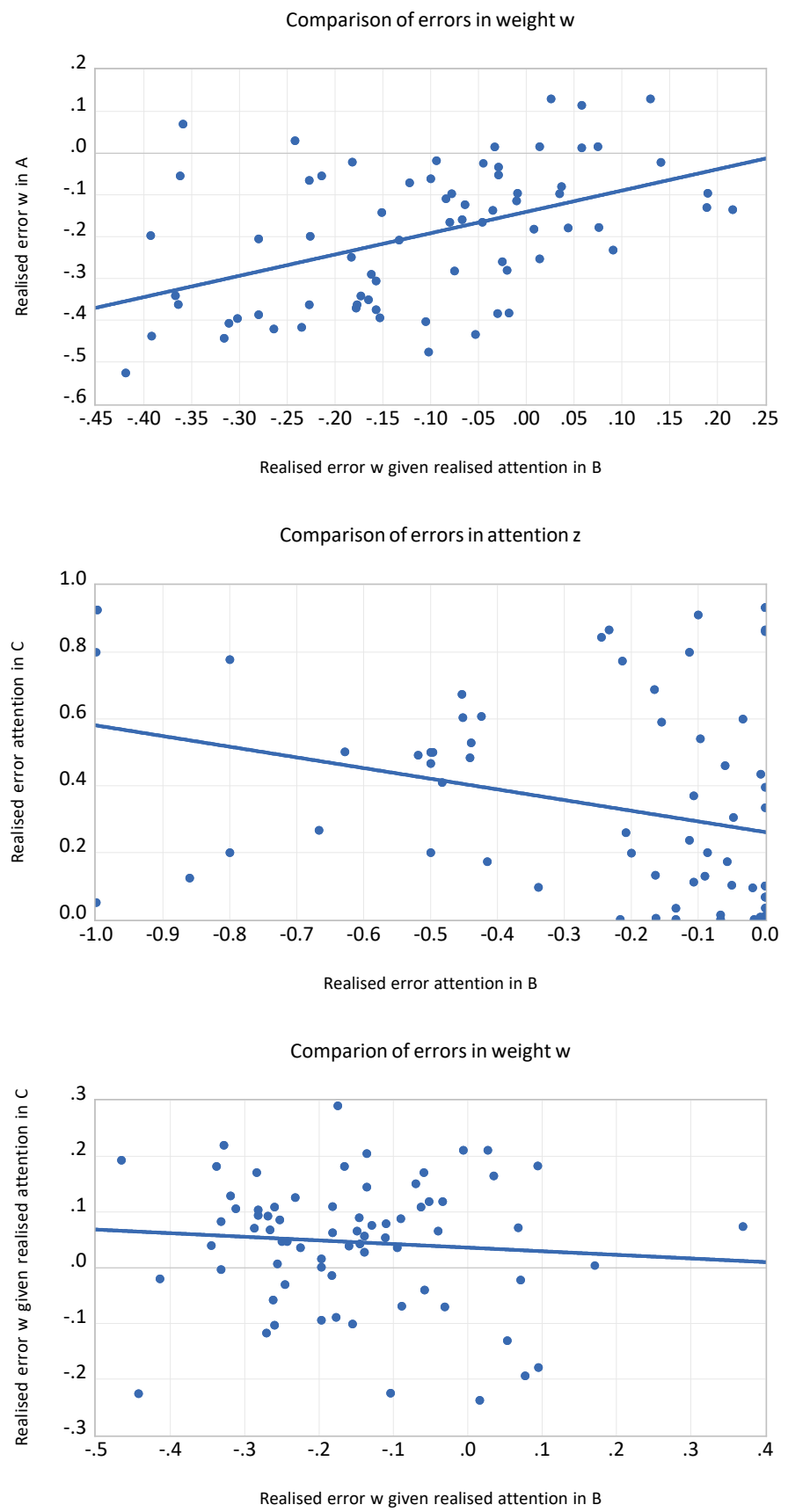

Figure 14: Comparison of realised individual average errors between treatments

that participants tend to play similarly in B and in $\mathrm{A}$. Those who play a low $\omega$ in B also tend to play a low $\omega$ in $\mathrm{A}$. This is not surprising because, apart from the choice of attention in B, participants play the same beauty contest game in A and B.

The middle panel plots the average error of each participant in the realised attention level in B and in C. Because optimal attention is 1 in B and 0 in C, errors can only be negative in $\mathrm{B}$ and positive in $\mathrm{C}$. The negative regression line indicates that participants who choose near-optimal attention in B also tend to choose near-optimal attention in treatment C. Thus, although introducing strategic complementarities shifts optimal attention from 
0 to 1 , it does not seem to alter the problem of choosing optimal attention per se.

The lower panel plots the average error of each participant in the realised weight $\omega$ in $\mathrm{B}$ and in $\mathrm{C}$. The average error in the realised weight $\omega$ in $\mathrm{B}$ and in $\mathrm{C}$ is conditional on the realised individual attention level in each period. The regression line does not indicate a clear relationship between realised errors in B and in C. Participants who perform well in the beauty contest game do not necessarily perform well in the fundamental-estimation game.

Result 7 (a) Under strategic complementarities, participants who choose a near-optimal weight $\omega$ without information acquisition (treatment A) also tend to choose a near-optimal weight $\omega$ with information acquisition (treatment B). (b) Participants who choose a near-optimal attention allocation under strategic complementarities (treatment B) also tend to choose a near-optimal attention allocation without strategic complementarities (treatment C). (c) Participants who choose a near-optimal weight $\omega$ under strategic complementarities (treatment B) do not necessarily choose a near-optimal weight $\omega$ without strategic complementarities (treatment $C$ ).

\subsection{Limited levels of reasoning}

We have seen above that the realised attention allocation is mainly explained by a failure to account for the expectations of others. In the beauty contest literature, realised deviations from equilibrium are usually measured in terms of limited levels of reasoning, which captures the importance that agents attribute to coordinating with others. We derive the theoretical benchmark to analyse the limited levels of reasoning in our beauty contest with both action and attention before presenting how well this model captures the data.

\subsubsection{Equivalence between a limited number of iterations and a limited degree of strategic complementarities}

This section presents the best responses for limited levels of reasoning under treatment B (beauty contest game with information acquisition). The best responses under treatment A are a particular case when the attention level $z$ is exogenously set at 1 .

We define level-0 types as players who ignore the strategic component of their action. This corresponds to solving the beauty contest model with $\gamma^{0}=0$. The action for level-0 reasoning is therefore $a_{i}^{0}=\mathbb{E}_{i}(\theta)$. The action for level- 1 reasoning consists of playing the best response assuming that other players are playing the level-0. For any $k>0$, a level- $k$ type is playing the best response assuming that the other players are level- $k-1$ types. Suppose that all other players $-i$ assign a weight $\rho^{k-1}$ to their signal $x_{1,-i}$. The best 
response of player $i$ is

$$
\begin{aligned}
a_{i}^{k}= & (1-\gamma) \mathbb{E}_{i}(\theta)+\gamma \mathbb{E}_{i}\left(\bar{a}_{-i}\right) \\
= & (1-\gamma) \mathbb{E}_{i}(\theta)+\gamma\left[\rho^{k-1} \mathbb{E}_{i}\left(\bar{x}_{1}\right)+\left(1-\rho^{k-1}\right) \mathbb{E}_{i}\left(\bar{x}_{2}\right)\right] \\
= & x_{1, i} \underbrace{\left[(1-\gamma) f+\gamma \rho^{k-1} \Omega_{1}+\gamma\left(1-\rho^{k-1}\right) \Omega_{2}\right]}_{\rho^{k}} \\
& +x_{2, i}\left[(1-\gamma)(1-f)+\gamma \rho^{k-1}\left(1-\Omega_{1}\right)+\gamma\left(1-\rho^{k-1}\right)\left(1-\Omega_{2}\right)\right]
\end{aligned}
$$

By identification, the level- $k$ best response, $\rho^{k}$, is a function of $\rho^{k-1}$ and the attention level $z_{i}$

$$
\rho^{k}=\frac{(1-\gamma) \kappa_{2}^{2}+\xi_{2}^{2}+z_{i}+\gamma \rho^{k-1}\left(\kappa_{1}^{2}+\kappa_{2}^{2}\right)}{\kappa_{1}^{2}+\kappa_{2}^{2}+\xi_{1}^{2}+\xi_{2}^{2}+1}
$$

Table 4 shows the iteration of the best response $\rho^{k}$ from level 0 to $\infty$ of reasoning and the attention level $z^{k}$ that maximises utility. Ignoring strategic complementarities, the optimal action is $\rho^{0}=0.25$ and the optimal attention $z^{0}=0$. As the number of iterations raises towards infinity, the strategy converges towards the suboptimal equilibrium $\left\{z^{\infty}=\right.$ $\left.0, \rho^{\infty}=0.435\right\}$. As explained in subsection 4.2, optimal attention is $z=0$ when the average attention of others is below $2.25 \%$. Since $z=0$ is optimal at the level-0 iteration, optimal attention remains $z=0$ as the number of iterations rises, leading players into the suboptimal equilibrium.

However, in the experiment, realised average attention is always significantly above $2.25 \%$. When the average attention of others is above this threshold, the optimal attention allocation is $z=1$. Applying the iteration process starting with an attention level of $z^{0}=1$ - though it is not optimal at level-0 reasoning - yields values of $\rho^{k}$ that converge towards the optimal equilibrium $\left\{z^{\infty}=1, \rho^{\infty}=0.870\right\}$. These values are reported in lines 6 and 7 of Table 4.

Applying the iteration process of best responses as above presents a shortcoming in our model due to the existence of two equilibria: as the number of iterations rises from 0 onwards, the solution converges to the suboptimal equilibrium. Convergence to the optimal equilibrium is obtained when starting with attention $z=1$, which is not optimal at level-0. However, an alternative formulation of limited levels of reasoning allows the creation of a gateway from level-0 with an optimal attention level $z=0$ to higher levels with an optimal attention level $z=1$.

When players operate a limited number of iterations, each iteration is operated with the actual degree of strategic complementarities $\gamma$. This response can be transposed into an equilibrium action (i.e., $\infty$ number of iterations) with a corresponding limited degree of strategic complementarities, $\gamma^{k}$. Instead of saying that players operate a finite number of iterations $k$ with the actual degree of strategic complementarities $\gamma$, one can transpose limited levels of reasoning by saying that players operate an infinite number of iterations 


\begin{tabular}{|c|l|c|c|c|c|c|c|c|}
\hline \multicolumn{2}{|c|}{ Attention at level-0 $z^{0}=0$ : convergence to the suboptimal equilibrium } \\
\hline & Level of reasoning $k$ & 0 & 1 & 2 & 3 & 4 & 5 & infinity \\
\hline 1 & $\rho^{k}$ & 0.250 & 0.356 & 0.401 & 0.421 & 0.429 & 0.432 & 0.435 \\
\hline 2 & $z^{k}$ & 0 & 0 & 0 & 0 & 0 & 0 & 0 \\
\hline 3 & $\gamma^{k}$ & 0 & 0.596 & 0.754 & 0.811 & 0.834 & 0.843 & 0.850 \\
\hline 4 & Expected utility & $\mathbf{- 0 . 7 5 0}$ & -0.657 & -0.602 & -0.580 & -0.571 & -0.568 & -0.565 \\
\hline 5 & $\sigma^{k}$ & - & 0.375 & 0.428 & 0.451 & 0.461 & 0.465 & 0.468 \\
\hline
\end{tabular}

\begin{tabular}{|c|l|c|c|c|c|c|c|c|}
\hline \multicolumn{9}{|c|}{ Attention at level-0 $z^{0}=1$ : convergence to the optimal equilibrium } \\
\hline & Level of reasoning $k$ & 0 & 1 & 2 & 3 & 4 & 5 & infinity \\
\hline 6 & $\rho^{k}$ & 0.500 & 0.713 & 0.803 & 0.841 & 0.858 & 0.864 & 0.870 \\
\hline 7 & $z^{k}$ & 1 & 1 & 1 & 1 & 1 & 1 & 1 \\
\hline 8 & $\gamma^{k}$ & 0 & 0.596 & 0.754 & 0.811 & 0.834 & 0.843 & 0.850 \\
\hline 9 & Expected utility & -1.000 & $\mathbf{- 0 . 6 2 9}$ & $\mathbf{- 0 . 4 0 7}$ & $\mathbf{- 0 . 3 2 0}$ & $\mathbf{- 0 . 2 8 5}$ & $\mathbf{- 0 . 2 7 1}$ & $\mathbf{- 0 . 2 6 1}$ \\
\hline 10 & $o^{k}$ & - & 0.750 & 0.856 & 0.901 & 0.921 & 0.929 & 0.935 \\
\hline
\end{tabular}

Table 4: Solutions for limited levels of reasoning

with a limited degree of strategic complementarities $\gamma^{k}$. From the equilibrium (level- $\infty$ ) action (9), we can compute the degree of strategic complementarity $\gamma^{k}$ that yields $\rho^{k}$

$$
\rho^{k}=\frac{\left(1-\gamma^{k}\right) \kappa_{2}^{2}+\xi_{2}^{2}+z_{i}}{\left(1-\gamma^{k}\right)\left(\kappa_{1}^{2}+\kappa_{2}^{2}\right)+\xi_{2}^{2}+\xi_{1}^{2}+1}
$$

which is equivalent to

$$
\gamma^{k}=\frac{\rho^{k}\left(\kappa_{1}^{2}+\kappa_{2}^{2}+\xi_{2}^{2}+\xi_{1}^{2}+1\right)-\left(\kappa_{2}^{2}+\xi_{2}^{2}+z_{i}\right)}{\rho^{k}\left(\kappa_{1}^{2}+\kappa_{2}^{2}\right)-\kappa_{2}^{2}}
$$

The values of $\gamma^{k}$ for each number of iterations and both levels of attention are reported in Table 4 on lines 3 and 8 . The limited degree of strategic complementarities $\gamma^{k}$ that corresponds to any level- $k$ of reasoning is the same regardless of the value of attention $z$.

Interpreting limited levels of reasoning in terms of a limited degree of strategic complementarities $\gamma^{k}$ (instead of a limited number of iterations of best responses) allows for the selection of the best strategy at any level- $k$. At level- $0, \gamma^{0}=0$, and the best strategy is $\left\{z^{0}=0, \rho^{0}=0.25\right\}$. At level-1, $\gamma^{1}=0.596$, and the best strategy is $\left\{z^{1}=1, \rho^{1}=0.713\right\}$. At level-2, $\gamma^{2}=0.754$, and the best strategy is $\left\{z^{2}=1, \rho^{2}=0.803\right\}$, and so on. The best attention allocation shifts from 0 to 1 between level- 0 and level- 1 reasoning.

\subsubsection{Extracting level- $k$ from individual decisions}

In treatment $\mathrm{B}$, given the realised weight $\omega_{i}$ and realised attention level $z_{i}$, we can compute the corresponding $\gamma^{i}$ for each individual decision. Because the theoretical $\gamma^{k}$ is independent of $z$, as depicted in Table 4, we can compare the realised $\gamma^{i}$ to the theoretical $\gamma^{k}$ and compute the level- $k$ iteration that each decision corresponds to.

The distribution of limited levels of reasoning is presented in Figure 15 for each treat- 
Level-k of reasoning in treatment $A$
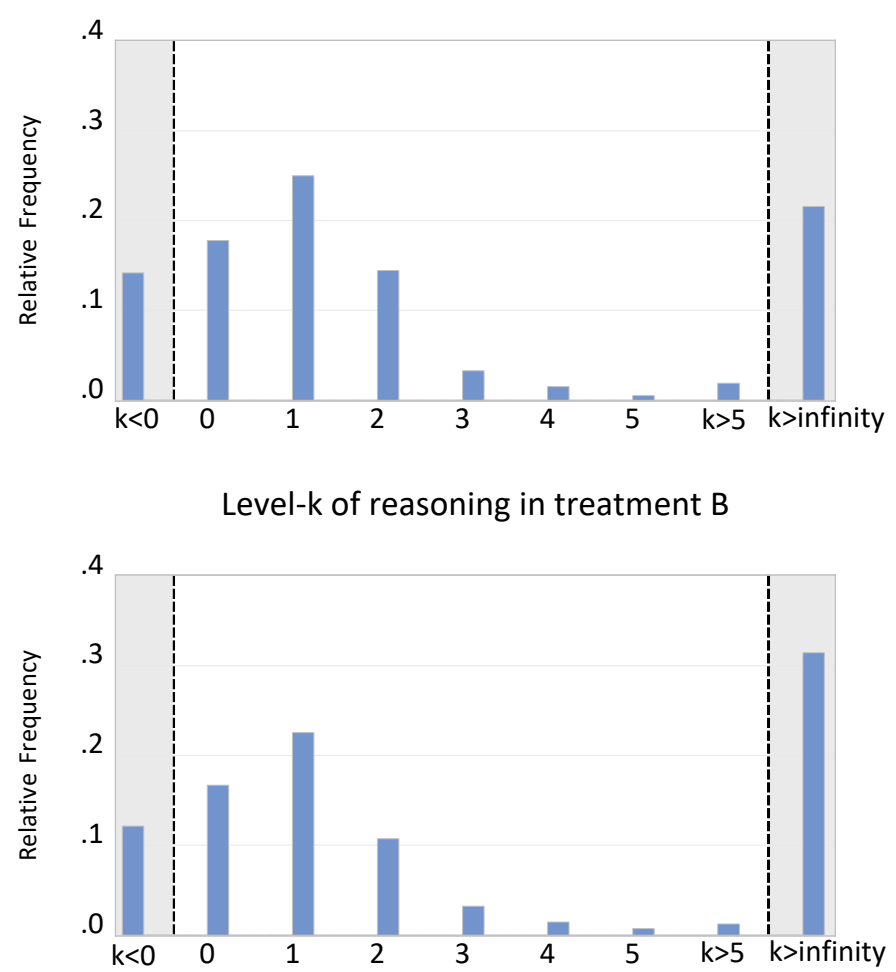

Level-k of reasoning in treatment $\mathrm{C}$

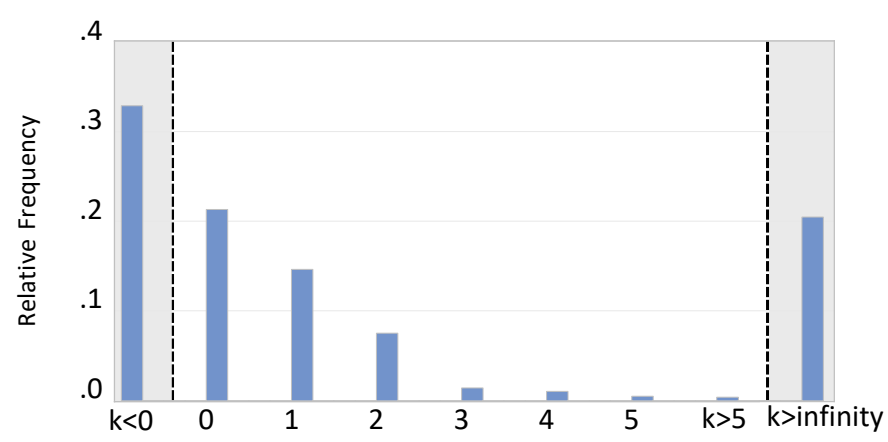

Figure 15: Distribution of limited levels of reasoning

ment. In treatment A, level-1 represents the most played strategy, which is in line with Baeriswyl and Cornand (2014) and Cornand and Heinemann (2014) $)^{9}$ but slightly below the level found by Nagel (1995) in a pure guessing game. The findings for treatment B are similar to those for treatment A except for the fact that the number of decisions above level- $\infty$ is higher, which is due to suboptimally low attention: a given action corresponds to a higher level of reasoning when attention is suboptimally low (as is often the case in B) than when it is optimally high (as is always the case in A). In treatments A and B, the presence of level-0 players - for whom it is optimal to play $z=0$, as explained above - induced lower values of $z$, partly explaining the deviation of average attention from equilibrium.

\footnotetext{
${ }^{9}$ Note that the definition of the level-0 type in our paper corresponds to their definition of the level-1 type.
} 
Treatment $\mathrm{C}$ contrasts with treatments $\mathrm{A}$ and $\mathrm{B}$. The majority of decisions correspond to level-0 or even levels below 0 . Nevertheless, although the fundamental-estimation game exhibits no strategic complementarities, a significant share of the decisions correspond to levels of reasoning above zero.

Result 8 (a) Decisions exhibit significant heterogeneity within and between treatments in their corresponding limited levels of reasoning. (b) Higher strategic complementarities lead to a higher level-k. (c) In treatment B, a large proportion of level- $k<1$ players rationalise a suboptimal average attention allocation below equilibrium; in treatment $C$, a large proportion of level- $k>0$ players rationalise a suboptimal average attention allocation above equilibrium.

\section{Discussion and conclusion}

Central bank communication aims to shape public expectations of key macroeconomic factors and to align private sector expectations with the actions of the central bank, enhancing monetary policy effectiveness. The market reaction to disclosures depends as much on how the central bank discloses information as on how much attention market participants give to the disclosures. In a beauty contest model with information acquisition by private agents, strategic complementarities give rise to a double overreaction mechanism to public disclosures. While strategic complementarities induce agents to give more weight in their action to the most common and least private signal than what would be justified by the signal's face value, these complementarities also induce agents to give more attention to this signal, further strengthening its weight in their actions. These theoretical predictions are supported by a laboratory experiment, although the double overreaction is weaker than predicted due to a lower effect of strategic complementarities on both the allocation of attention and the beauty contest action.

As an attention level that is weaker than theoretically predicted explains to a large extent the weaker focal potential of public disclosures (i.e., a weaker overreaction than that theoretically predicted), such limited attention might usefully be accounted for in designing optimal central bank communication. Though relying on an abstract model, our experiment captures realistic features of the way central banks may communicate in practice. In particular, it offers a rationale for the so-called forward guidance puzzle, i.e., the fact that macroeconomic responses to forward guidance are much mitigated in comparison to the theoretical predictions of standard new Keynesian models. Indeed, the experimentally observed weaker attention to common information may rationalise why public disclosures do not fully drive market actions in the direction of announcements. Among the various alternative belief-based interpretations of the forward guidance puzzle, Angeletos and Lian (2018) attribute it to a lack of common knowledge and, Garcia-Schmidt and Woodford (2018) and Farhi and Werning (2017) to limited level-k reasoning. If the forward guidance puzzle is explained by a lack of private sector attention, as our experimental results may suggest, central banks should consider how to increase attention. Improving attention 
operates through model parameters that are not under the control of the central bank in the present model. This improvement would involve, for example, better control of the timing and channels of communication (less frequent to focus attention or more frequent to reduce it, depending on the context) or increasing public economic literacy to raise awareness of what monetary policy and forward guidance in particular are all about.

\section{References}

Angeletos, G.-M. and Lian, C. (2018). Forward guidance without common knowledge. American Economic Review, 108(9):2477-2512.

Angeletos, G.-M. and Pavan, A. (2007). Efficient use of information and social value of information. Econometrica, 75(4):1103-1142.

Baeriswyl, R. and Cornand, C. (2014). Reducing overreaction to central banks' disclosures: theory and experiment. Journal of the European Economic Association, 12(4):10871126.

Caplin, A. and Dean, M. (2013). Behavioral implications of rational inattention with shannon entropy. NBER Working Paper No. 19318.

Caplin, A. and Dean, M. (2015). Revealed preference, rational inattention, and costly information acquisition. American Economic Review, 105(7):2183-2203.

Chahrour, R. (2014). Public communication and information acquisition. American Economic Journal: Macroeconomics, 6(3):73-101.

Cheremukhin, A., Popova, A., and Tutino, A. (2015). A theory of descrete choice with information costs. Journal of Economic Behavior and Organization, 113:34-50.

Colombo, L., Femminis, G., and Pavan, A. (2014). Information acquisition and welfare. Review of Economic Studies, 81(4):1438-1483.

Cornand, C. and Heinemann, F. (2014). Measuring agents' overreaction to public information in games with strategic complementarities. Experimental Economics, 17(1):61-77.

Del Negro, M., Giannoni, M., and Patterson, C. (2015). The forward guidance puzzle. Staff Reports, 547.

Farhi, E. and Werning, I. (2017). Monetary policy, bounded rationality, and incomplete markets. NBER Working Paper No. 23281.

Garcia-Schmidt, M. and Woodford, M. (2018). Are low interest rates deflationary? a paradox of perfect-foresight analysis. American Economic Review (forthcoming).

Hellwig, C. and Veldkamp, L. (2009). Knowing what others know: Coordination motives in information acquisition. Review of Economic Studies, 76:223-251. 
Llosa, L. G. and Venkateswaran, V. (2013). Efficiency with endogenous information choice. unpublished manuscript.

Mackowiak, B. and Wiederholt, M. (2015). Business cycle dynamics under rational inattention. Review of Economic Studies, 82(4):1502-1532.

Morris, S. and Shin, H. S. (2002). Social value of public information. American Economic Review, 92(5):1521-1534.

Myatt, D. P. and Wallace, C. (2012). Endogenous information acquisition in coordination games. Review of Economic Studies, 79:340-374.

Myatt, D. P. and Wallace, C. (2014). Central bank communication design in a lucas-phelps economy. Journal of Monetary Economics, 63:64-79.

Nagel, R. (1995). Unraveling in guessing games: An experimental study. American Economic Review, 85:1313-1326.

Pavan, A. (2016). Attention, coordination, and bounded recall. unpublished manuscript.

Sims, C. A. (2003). Implications of rational inattention. Journal of Monetary Economics, 50(3):665-690.

Szkup, M. and Trevino, I. (2017). Sentiments, strategic uncertainty, and information structures in coordination games. unpublished manuscript.

Woodford, M. (2005). Central bank communication and policy effectiveness. NBER Working Paper, (11898). 


\section{A Payoff sensitivity}

Figures 16 and 17 illustrate the sensitivity of the payoffs in treatments B and C to deviations from optimal attention and optimal action. The upper graph in figure 16 shows for treatment B the expected utility as a function of $z$ when $\omega$ remains at its equilibrium value $\omega=0.870$ (solid black line) and when $\omega$ adjusts to its optimal value relative to $z$ (dotted blue line). The lower graph shows the expected utility as a function of $\omega$ when $z$ remains at its equilibrium value $z=1$ (solid black line) and when $z$ adjusts to its optimal value relative to $\omega$ (dotted blue line). The thick lines plot utility when all agents deviate from the optimum value; the thin lines plot utility when only one agent deviates.

Figure 17 shows the utility as a function of $z$ (upper graph) and $\omega$ (lower graph) for treatment C. Without strategic complementarities, deviations from the optimal weight $\omega$ by all agents or by only one agent yield the same utility.
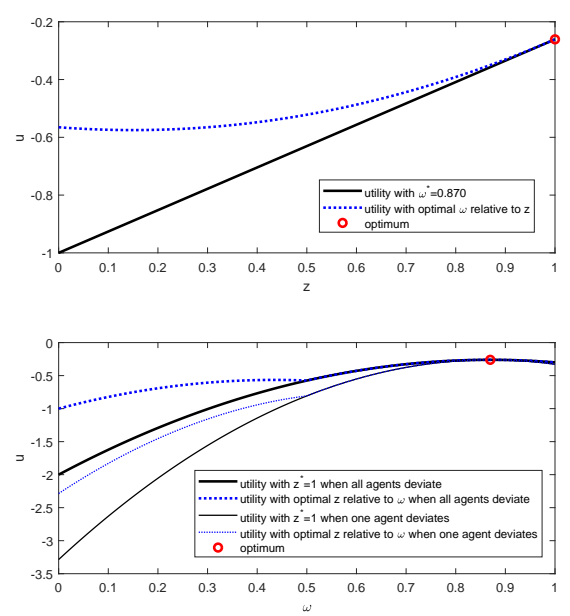

Figure 16: Utility deviation for treatment B
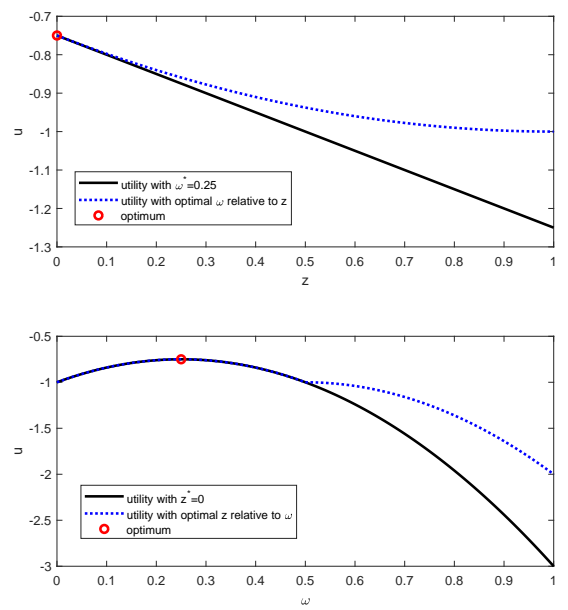

Figure 17: Utility deviation for treatment C 


\section{B Additional figures}

Treatment B

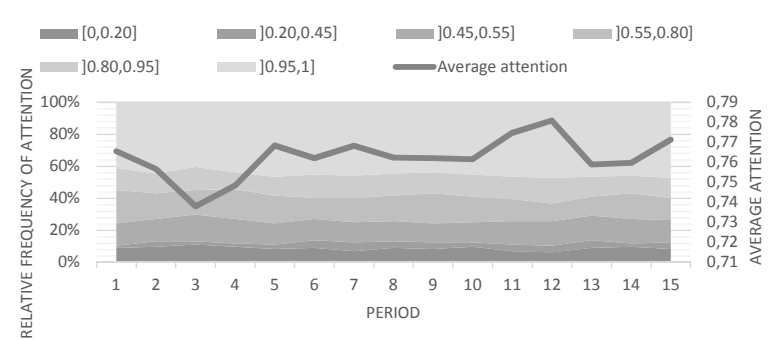

Treatment C

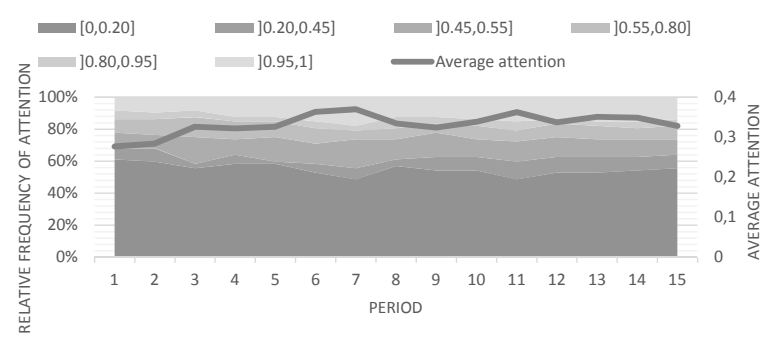

Treatment B

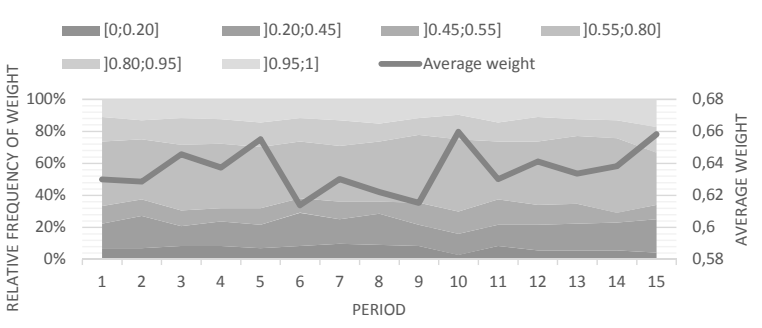

Treatment A

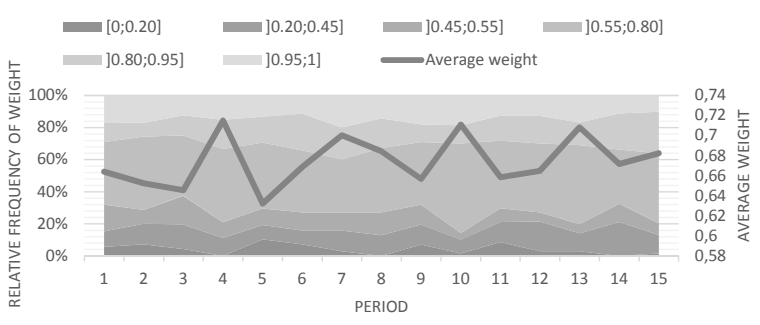

Figure 18: Convergence

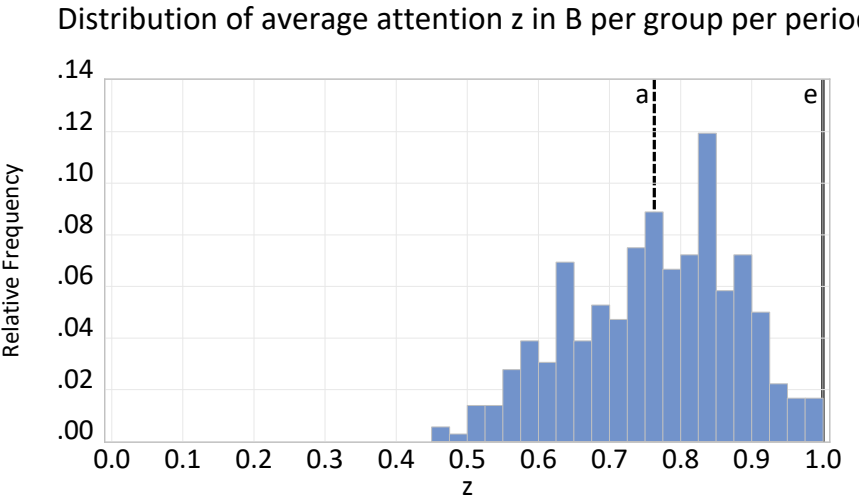

Distribution of average attention $\mathrm{z}$ in $\mathrm{C}$ per group per period

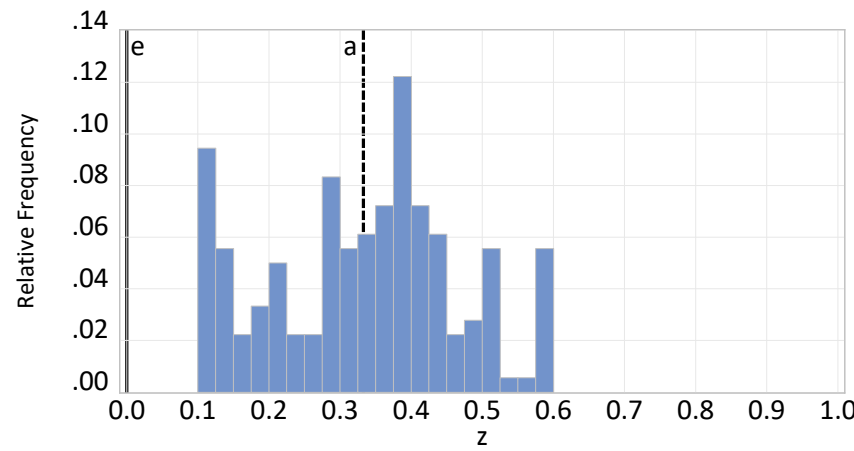

Figure 19: Distribution of average attention given to $x_{1, i}$ within each group in every period 


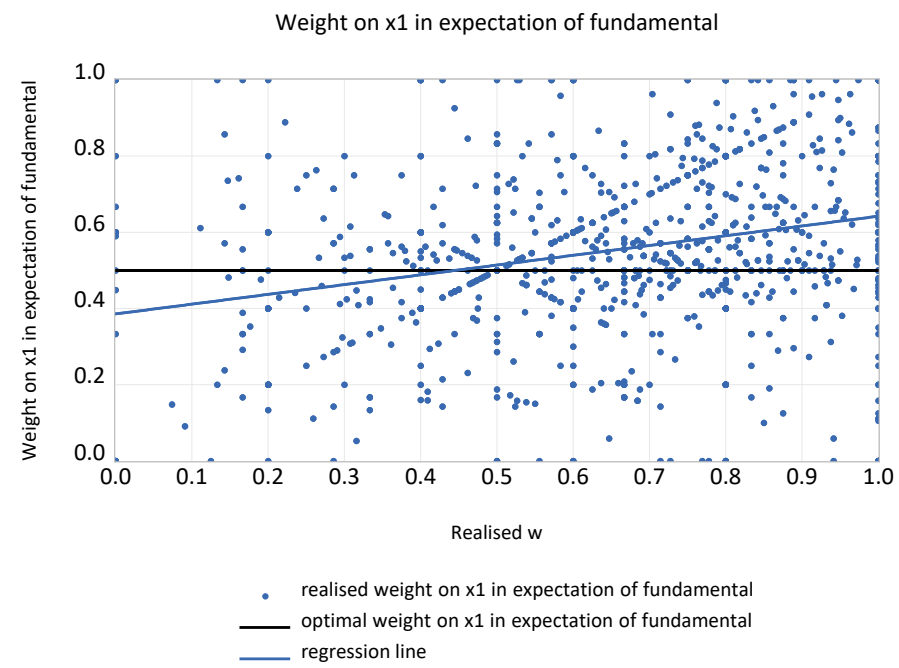

(a) Weight assigned to $x_{1, i}$ in the expectation of the fundamental as a function of realised $\omega$ in treatment $\mathrm{A}$

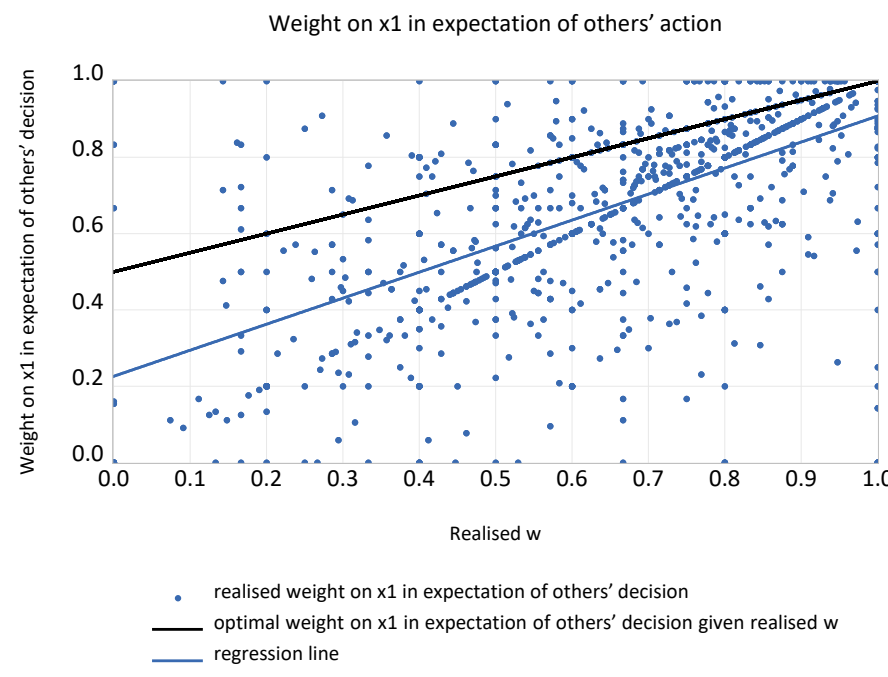

(b) Weight assigned to $x_{1, i}$ in the expectation of others' action as a function of realised $\omega$ in treatment $\mathrm{A}$

Figure 20: Effect of realised $\omega$ on expectations' formation in treatment A 


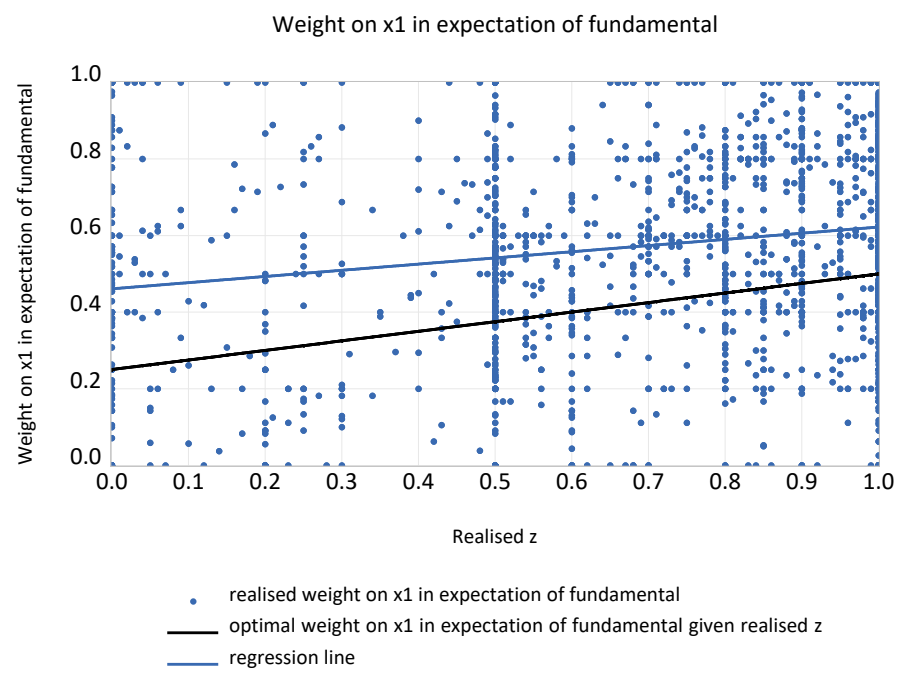

(a) Weight assigned to $x_{1}$ in the expectation of the fundamental as a function of realised $z$ in treatment $\mathrm{B}$

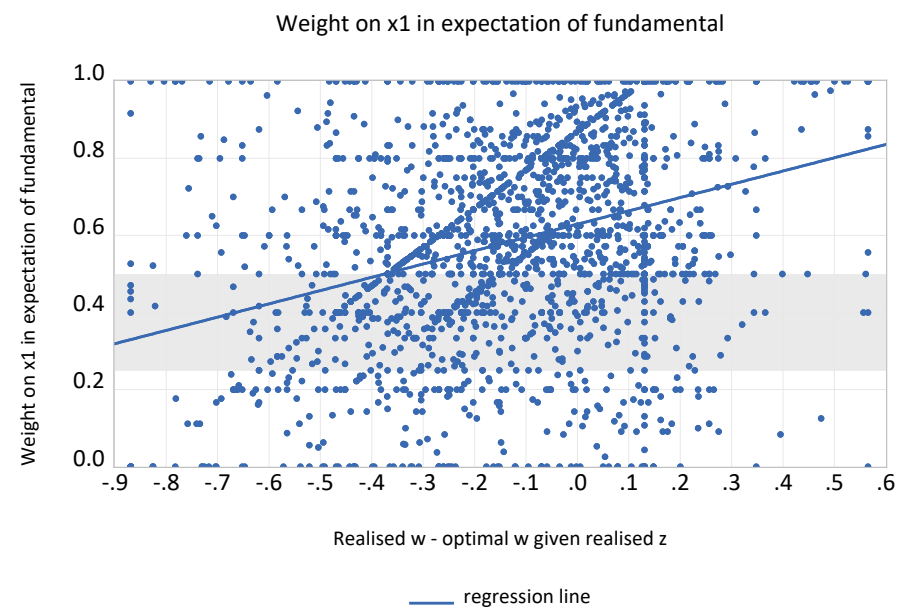

(b) Weight assigned to $x_{1}$ in the expectation of the fundamental as a function of difference between realised $\omega$ and optimal $\omega$ given realised $z$ in treatment $\mathrm{B}$

Figure 21: Weight assigned to $x_{1}$ in the expectation of the fundamental in treatment B 


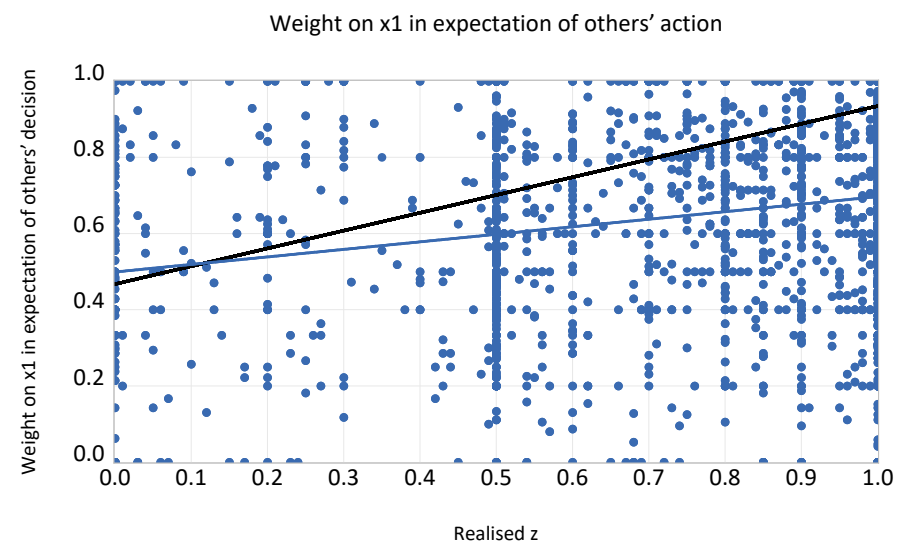

realised weight on $x 1$ in expectation of others decision
optimal weight on $x 1$ in expectation of others decision given realised $z$
regression line

(a) Weight assigned to $x_{1}$ in the expectation of others' decision as a function of realised $z$ in treatment $\mathrm{B}$

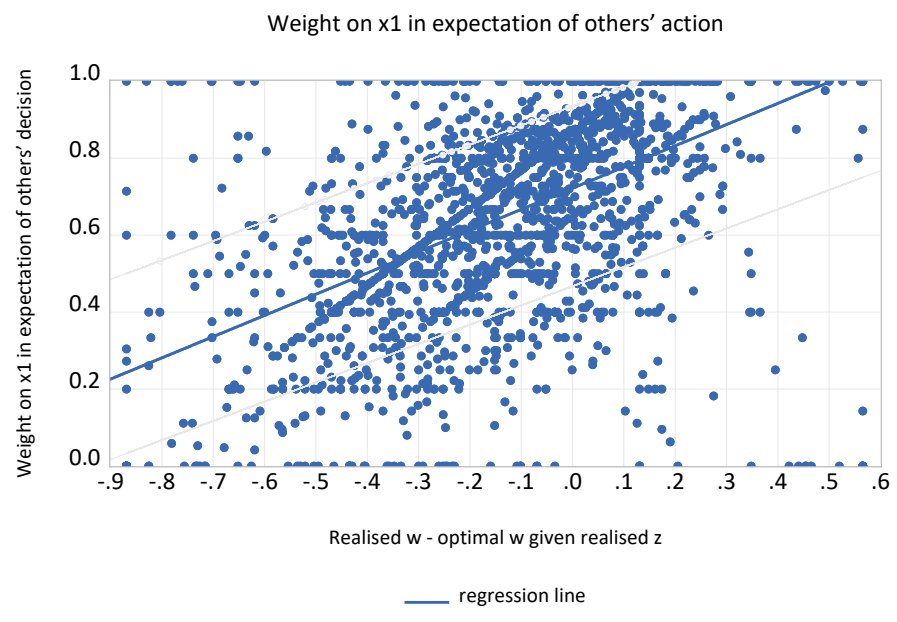

(b) Weight assigned to $x_{1}$ in the expectation of others' decision as a function of difference between realised $\omega$ and optimal $\omega$ given realised $z$ in treatment $\mathrm{B}$

Figure 22: Weight assigned to $x_{1}$ in the expectation of others' decision in treatment $\mathrm{B}$ 


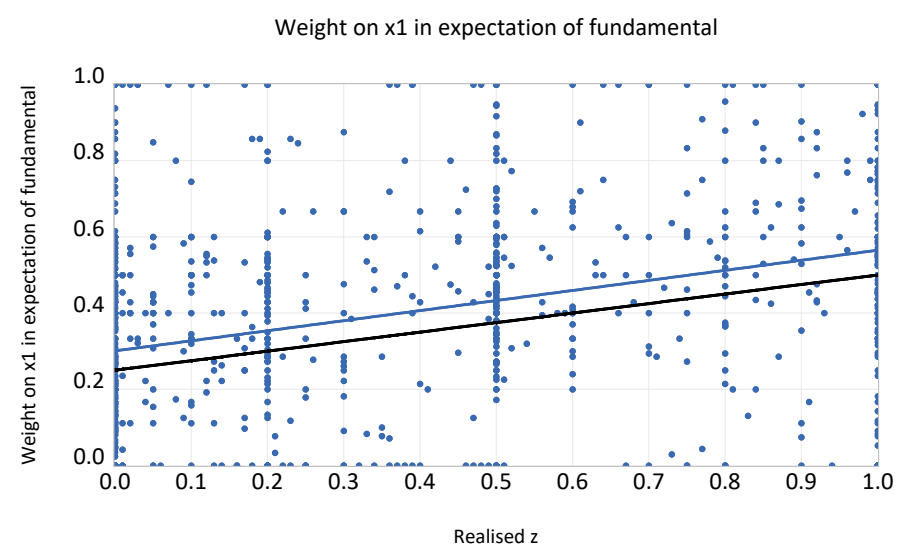

- realised weight on $x 1$ in expectation of fundamental

optimal weight on $\mathrm{x} 1$ in expectation of fundamental given realised $\mathrm{z}$

regression line

(a) Weight assigned to $x_{1}$ in the expectation of the fundamental as a function of realised $z$ in treatment $\mathrm{C}$

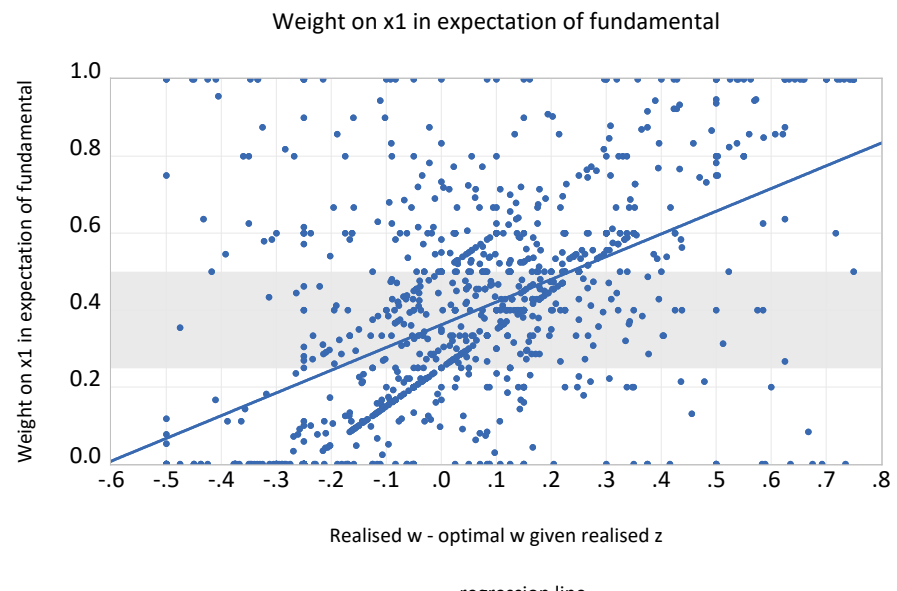

(b) Weight assigned to $x_{1}$ in the expectation of the fundamental as a function of difference between realised $\omega$ and optimal $\omega$ given realised $z$ in treatment $\mathrm{C}$

Figure 23: Weight assigned to $x_{1}$ in the expectation of the fundamental in treatment $\mathrm{C}$ 


\section{Translation of instructions}

Instructions to participants varied according to the sessions. We present the instructions for sessions 1 and 2 with treatments A and B. For the other sessions, the instructions were adapted accordingly and are available upon request. ${ }^{10}$

\section{INSTRUCTIONS}

\section{Hello and welcome to our laboratory.}

During the experiment in which you will participate, you will be asked to make decisions. These instructions explain what these decisions are and how your decisions will earn you a certain amount of money.

The earnings you receive during this experiment will depend partly on your own decisions, partly on the decisions of the other participants, and partly on chance through random draws. All your decisions will be processed anonymously, and you will never have to enter your name into the computer. The amount of money earned during the experiment will be paid to you individually at the end of the experiment.

You are one of 18 people participating in this experiment, and you are all divided into three groups of 6 people. These three groups are totally independent and will not interact with each other throughout the experiment. Each participant interacts only with the other participants in his or her group. These instructions describe the rules of the game for a group of 6 participants and are the same for all participants.

At the end of this reading, feel free to ask questions if you wish.

\section{General framework of the experiment}

The experiment includes 2 games in total. Each game is repeated for 15 periods so that the experiment has a total of 30 periods. During each of these 30 periods, the decisions you make will determine your earnings. During the experiment, your earnings are expressed in ECU. At the end of the experiment, the ECUs you earn will be converted into euros. The conversion rate is $100 \mathrm{ECU}=12$ euros.

Note that there are a total of 15 periods in a game, but only 2 periods will be randomly selected to calculate your payoff. Since all periods are equally likely to be chosen for the calculation of your payoff, you must give equal weight to each of the 15 periods. At the end of the experiment, a participant will randomly draw 2 periods from each game, which will serve as the basis for your payoff.

\section{Rule determining the payoff in each of the 30 periods}

At the beginning of each period, the computer draws a positive integer Z. You will not know the true value of $\mathbf{Z}$. This number $\mathbf{Z}$ is different in each period but identical for all participants in the same group.

\footnotetext{
${ }^{10}$ What follows is a translation (from French to English) of the instructions given to the participants.
} 
In each period, you are asked to make a decision by choosing a number $\mathbf{D}$. Decision $\mathbf{D}$ will determine the ECU payoff of each participant for each period according to the formula:

$$
50-1.5(\mathbf{D}-\mathbf{Z})^{2}-8.5(\mathbf{D}-\text { average of decisions } \mathbf{D} \text { of the other participants })^{2}
$$

This formula indicates that your payoff is higher the closer your decision $\mathbf{D}$ is,

- on the one hand, to the unknown number $\mathbf{Z}$ and

- on the other hand, to the average decision $\mathbf{D}$ of the other participants in your group.

To maximise your payoff, you must make a decision $\mathbf{D}$ that is close to both the unknown number $\mathbf{Z}$ and the average of the decisions $\mathbf{D}$ of the other participants. Note, however, that it is more important to be close to the average decision of the other participants than to the unknown number $\mathbf{Z}$.

No participant knows the true value of $\mathbf{Z}$ when making his or her decision. However, to help you in your choice of $\mathbf{D}$, you can observe two hints on the unknown number $\mathbf{Z}$ as explained below.

\section{Your hints on $\mathrm{Z}$ in the first game (periods 1 to 15 )}

For each period of the first game, you will have two hints (numbers) on the unknown number $\mathbf{Z}$. These hints contain unknown errors.

\section{- Common hint $\mathbf{X}$}

In each period, you, as well as all the other members of your group, will receive a common hint $\mathbf{X}$ on the unknown number $\mathbf{Z}$. This common hint is centred on $\mathbf{Z}$ and contains an error randomly selected from a normal distribution of mean 0 and variance 2 . The variance of a random variable measures the dispersion of that variable around its mean. It is the inverse of precision; the higher the variance, the lower is the precision, and vice versa. This common hint $\mathbf{X}$ is the same for all participants.

\section{- Private hint $\mathbf{Y}$}

In addition to this common hint $\mathbf{X}$, in each period, each participant receives a private hint $\mathbf{Y}$ on the unknown number $\mathbf{Z}$. Each private hint is also centred on $\mathbf{Z}$ and contains an error randomly selected from a normal distribution of mean 0 and variance 2 . Your private hint and the private hints of each of the other participants are selected independently of each other, so that each participant will receive a different private hint from that of the other participants.

\section{Distinction between common hint $\mathrm{X}$ and private hint $\mathrm{Y}$}

The common hint $\mathbf{X}$ and your private hint $\mathbf{Y}$ have the same accuracy (i.e., the same variance of 2): both hints are on average equally informative about the unknown number Z. The only distinction between the two hints is that each participant observes a private 
hint $\mathbf{Y}$ different from that of the other participants, while all participants observe the same common hint $\mathbf{X}$.

\section{How to make your decision?}

Since you do not know the errors in your hints, it is natural to choose as decision $\mathbf{D}$ a number between the common hint $\mathbf{X}$ and your private hint $\mathbf{Y}$ if $\mathbf{X}$ is less than $\mathbf{Y}$ and between $\mathbf{Y}$ and $\mathbf{X}$ otherwise. To make your decision, we ask you to select a number between the common hint $\mathbf{X}$ and your private hint $\mathbf{Y}$ using a cursor. You must therefore choose how to combine your two hints in order to maximise your payoff. Note, however, that the payoff formula indicates that it is more important that your decision $\mathbf{D}$ is close to the average of the decisions of the other participants than that is close to the unknown number $\mathbf{Z}$.

To make it easier for you to choose $\mathbf{D}$, the formula for your payoff will appear on the screen and allow you to estimate your payoff based on your choice of $\mathbf{D}$ and, your estimate of the unknown number $\mathbf{Z}$ as well as your estimate of the average decision of the other participants. If the estimated payoff does not differ by more than 4 ECU from the actual payoff you have earned, you will receive a bonus of 10 ECU in addition to the payoff for the period.

Once you have determined your estimate of $\mathbf{Z}$ (EZ), your estimate of the decision of others (ED) and your decision D using the sliders, click on the "Validate" button. As soon as all participants have done the same, the period ends, and the result of the period is communicated to you. Then, the next period begins.

As soon as the 15 periods of the first game are completed, the second game of the experiment begins.

Note: If the payoff from your decision $\mathbf{D}$ in a period is negative, it will be reduced to 0 .

\section{Your hints on $\mathrm{Z}$ in the second game (periods 16 to 30 )}

As in the first game, you must make a decision $\mathbf{D}$ to maximise your payoff given by the formula on page 3 of the instructions. The second game is distinguished from the first by the fact that you do not directly observe any hints on $\mathbf{Z}$. However, you must choose the level of attention $\mathbf{A}$ with which you observe two hints on $\mathbf{Z}$. The attention you give to each of these hints determines the variance of the private error with which you observe these hints as described below.

In each period, you therefore have two choices to make: first, you must choose the level of attention $\mathbf{A}$ that you give to each of the hints $\mathbf{M}$ and $\mathbf{N}$; then, given hints $\mathbf{M}$ and $\mathbf{N}$ that you observe, you must make your decision $\mathbf{D}$ to maximise your payoff.

\section{Choice of A: allocation of your attention between the two hints $\mathrm{M}$ and $\mathrm{N}$}

You have a total attention of $100 \%$ that you must divide between the two hints $\mathbf{M}$ and $\mathbf{N}$. The amount of attention you give to hint $\mathbf{M}$ is denoted by $\mathbf{A}$ and is between $0 \%$ and 
$100 \%$. The amount of attention you give to hint $\mathbf{N}$ is equal to $1-\mathbf{A}$ and corresponds to the difference between the total attention $100 \%$ and the attention given to hint $\mathbf{M}$.

\section{- Hint $\mathbf{M}$}

Hint $\mathbf{M}$ contains an error common to all participants as well as a private error specific to each participant.

- An error common to all participants

Hint $\mathbf{M}$ is centred on $\mathbf{Z}$ and contains a common error randomly selected from a normal distribution of mean 0 and variance 2. This error term of hint $\mathbf{M}$ is common to all participants.

- Private error specific to each participant depending on the choice of $\mathbf{A}$

In addition to the common error, you observe hint $\mathbf{M}$ with a randomly selected private error from a normal distribution of mean 0 and variance 1-A. Thus, the greater the amount of attention $\mathbf{A}$ you pay to hint $\mathbf{M}$, the more you will observe hint $\mathbf{M}$ with a small private error. The private error of each participant is different from that of the other participants because the private errors are derived independently of each other from a normal distribution and because the variance of the normal distribution depends on the level of attention $\mathbf{A}$ that each participant has chosen.

\section{- Hint $\mathbf{N}$}

Hint $\mathbf{N}$ contains only one private error specific to each participant, and its common error for all participants is equal to 0 . Hint $\mathbf{N}$ is centred on $\mathbf{Z}$ and contains a private error randomly selected from a normal distribution of mean 0 and variance $1+\mathbf{A}$. Thus, the greater the attention $\mathbf{A}$ that you give to hint $\mathbf{M}$, the lower is the attention you give to hint $\mathbf{N}$ and the more you will observe hint $\mathbf{N}$ with a strong private error. The private error of each participant is different from that of the other participants because the private errors are derived independently of each other from a normal distribution and because the variance of the normal distribution depends on the level of attention $\mathbf{A}$ that each participant has chosen.

For example, as you can see on the graph below, if you choose a maximum level of attention A of $100 \%$, you will observe hint $\mathbf{M}$ without a private error $(1-100 \%=0)$ and hint $\mathbf{N}$ with a private error of variance $1+100 \%=2$. If, however, you choose a minimum level of attention $\mathbf{A}$ of $0 \%$, you will observe hint $\mathbf{M}$ with a private error of variance $1-0 \%=1$ and hint $\mathbf{N}$ with a private error of variance $1+0 \%=1$ as well.

In concrete terms, you must use the cursor to select the amount of attention $\mathbf{A}$ between $0 \%$ and $100 \%$ to give to hint $\mathbf{M}$. The attention you pay to hint $\mathbf{N}$ will automatically correspond to the difference between $\mathbf{1 0 0 \%}$ and the number $\mathbf{A}$ that you have selected using the cursor. The closer your $\mathbf{A}$ is to $100 \%$, the more attention you will pay to hint $\mathbf{M}$ (and the less to hint $\mathbf{N}$ ). Conversely, the closer your $\mathbf{A}$ is to $0 \%$, the more attention you will pay to hint $\mathbf{N}$ (and the less to hint $\mathbf{M}$ ). 


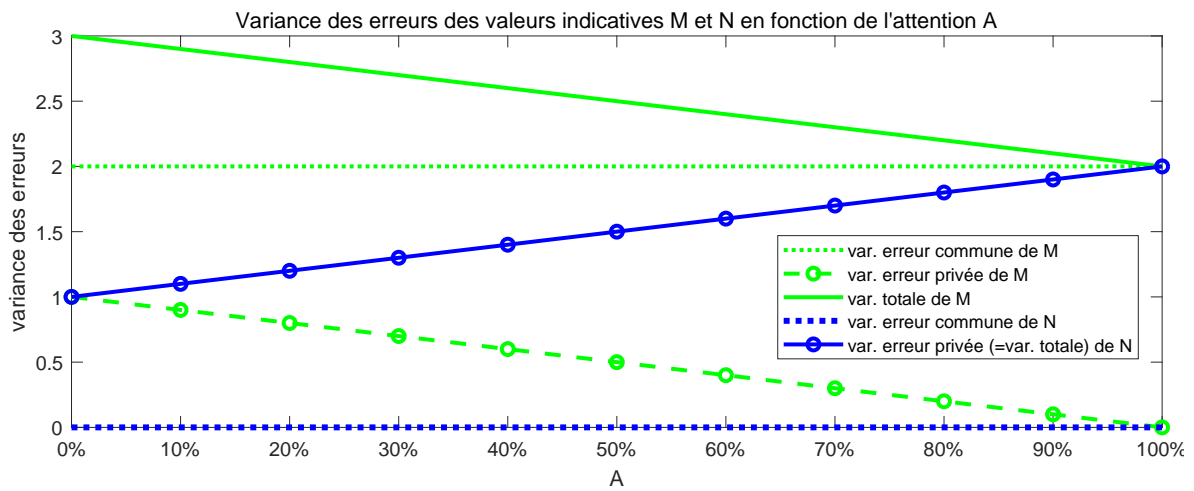

Once you have fixed the cursor on the decision A of your choice, click on the "Validate" button. As soon as all participants have done the same, the computer will randomly draw hints $\mathbf{M}$ and $\mathbf{N}$ according to the allocation of attention you have chosen. Hints $\mathbf{M}$ and $\mathbf{N}$ will then be displayed on your screen.

\section{Choice of D: decision determining your payoffs}

Since you do not know the errors in your hints, it is natural to choose as decision $\mathbf{D}$ a number between your hint $\mathbf{M}$ and your hint $\mathbf{N}$ if $\mathbf{M}$ is less than $\mathbf{N}$ and between $\mathbf{N}$ and $\mathbf{M}$ otherwise. To make your decision, we ask you to select a number between hint $\mathbf{M}$ and hint $\mathbf{N}$ using the cursor. You must therefore choose how to combine your two hints to maximise your payoff.

Note that the formula for payoffs on page 3 of the instructions indicates that it is more important that your decision $\mathbf{D}$ is close to the average of the decisions of the other participants than that it is close to the unknown number $\mathbf{Z}$.

Note also that by choosing A beforehand, you will have chosen the amount of attention you give to hint $\mathbf{M}$ relative to hint $\mathbf{N}$, which implies that you will have determined the variance of the private error of these hints. To maximise your payoff associated with your decision $\mathbf{D}$, you must take into account the choice of $\mathbf{A}$ you have made beforehand. Conversely, you must choose your $\mathbf{A}$ by considering its effect on the information you will have to make your decision $\mathbf{D}$.

To make it easier for you to choose $\mathbf{D}$, the formula for the payoffs will appear on the screen and allow you to estimate your payoff based on your choice of $\mathbf{D}$ and your estimate of the unknown number $\mathbf{Z}$ as well as your estimate of the average decision of the other participants. If the estimated payoff does not differ by more than 4 ECU from the actual payoff you have earned, you will receive a bonus of 10 ECU in addition to the payoff for the period.

Once you have determined your estimate of $\mathbf{Z}$ (EZ), your estimate of the decision of others (ED) and your decision D using the sliders, click on the "Validate" button. As soon as all participants have done the same, the period ends, and the result of the period is communicated to you. Then, the next period begins. 
As soon as the 15 periods of the second game are completed, the experiment ends.

Note: If the payoff from your decision $\mathbf{D}$ in a period is negative, it will be reduced to 0 . 


\section{Example of screens}

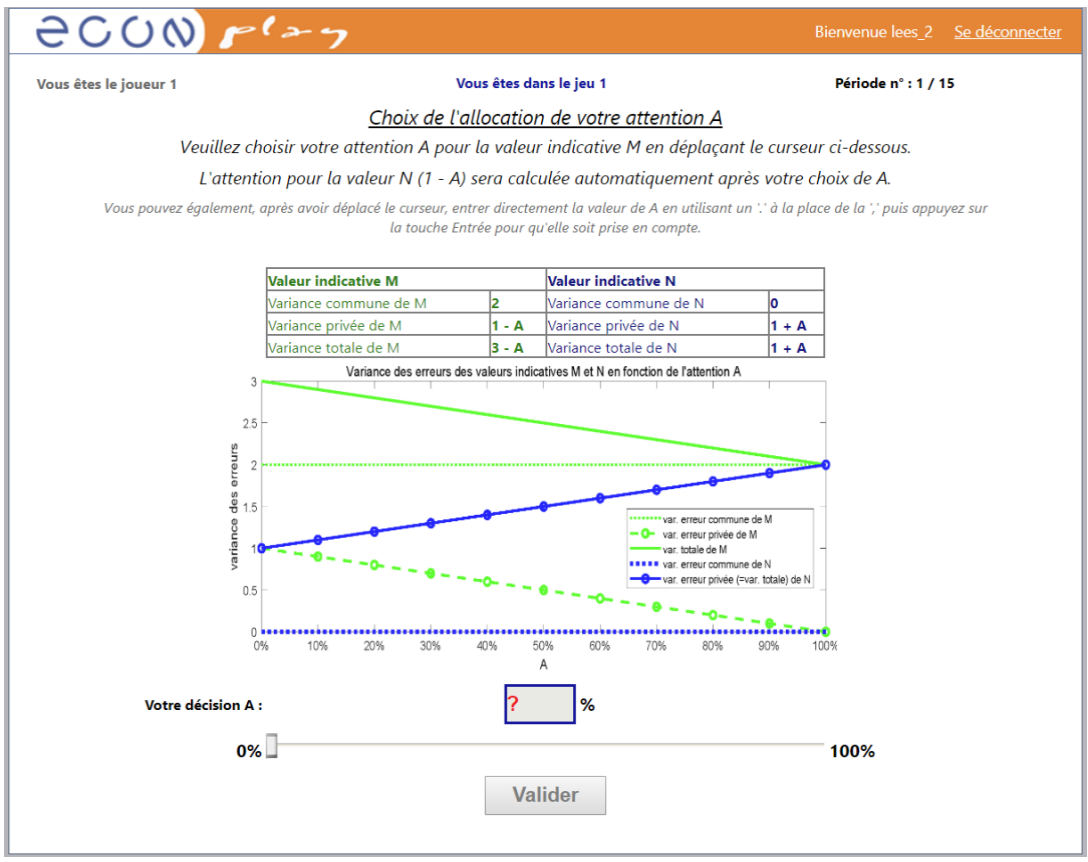

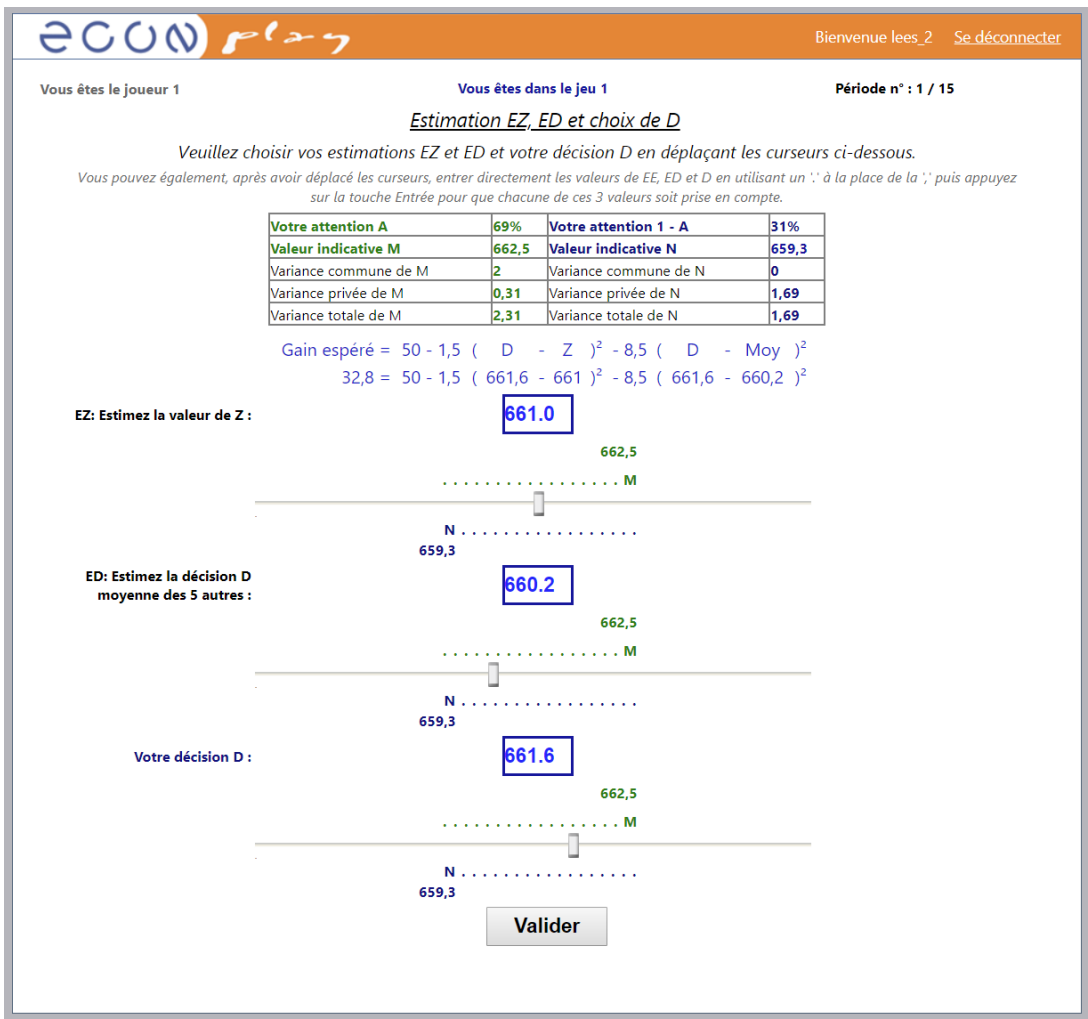


Jeu 1 - Résultats de la période $n^{\circ}: 1 / 15$

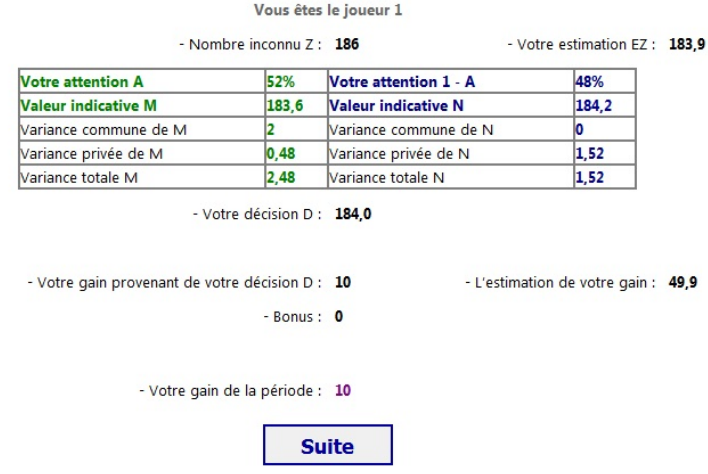

